\title{
Numerical Simulation of Flow over Bionic Airfoil
}

\author{
Lishu Hao $\mathbb{D}$, Yongwei Gao $\mathbb{D}$, Binbin Wei $\mathbb{D}$, and Ke Song \\ National Key Laboratory of Science and Technology on Aerodynamic Design and Research, Northwestern Polytechnical University, \\ Xi'an 710072, China
}

Correspondence should be addressed to Lishu Hao; haolishu@nwpu.edu.cn

Received 22 February 2021; Revised 25 June 2021; Accepted 18 August 2021; Published 13 September 2021

Academic Editor: Hongbing Ding

Copyright (c) 2021 Lishu Hao et al. This is an open access article distributed under the Creative Commons Attribution License, which permits unrestricted use, distribution, and reproduction in any medium, provided the original work is properly cited.

In this study, the aerodynamic performance of bionic airfoil was numerically studied by CFD method. The bionic airfoil was represented by the combination of airfoil and a small trailing edge flap. A variety of configurations were calculated to study the effect of flap parameters, such as the flap angle, position, and shape, on the bionic airfoil aerodynamic characteristics based on two layouts which were that (1) there was a tiny gap between the airfoil and the flap and (2) there was no gap between the two. The results showed that the flap angle and position had significant effects on the aerodynamic performance of the airfoil with the two layouts. Compared with the clean airfoil, the maximum lift coefficients of the first layout and the second layout could be increased by $10.9 \%$ and $7.9 \%$, respectively. And the effective angle of attack (AoA) range for improving the lift-todrag ratio could reach $7^{\circ}$. The flap shape also affected the airfoil aerodynamic characteristics, and the flap with the sinusoid curve shape showed ideal performance.

\section{Introduction}

Bionic morphing aircraft is a new type of aircraft with promising applications in military and civilian fields. An important part of this aircraft is the morphing wing, and a key research area is the bionic airfoil, which has gained worldwide attention.

Aldheeb et al. [1] presented an overview of prior analyses and experiments on the aerodynamic performance and mechanical properties of birds in steady nonflapping flight. Bechert et al. [2,3] reviewed the effects of biological surface structure and properties on fluid dynamics, and the paper focuses on techniques for both reducing wall shear stress and controlling boundary layer separation. Liu et al. [4] reviewed the biological fluid power systems and their potential bionic applications.

Research into the bionic wing mainly focuses on three topics: extracting bird wing shapes and analyzing their aerodynamic performance [5-7], achieving flexible deformations of a wing $[8,9]$, and observing the wing details of a flying bird to extract an airfoil model [10]. A photo of the landing bird is shown in Figure 1, and its behavior was explained by Liebe [11] from an aerodynamic perspective. Meyer [12] designed a bionic wing and verified it by fee flight tests.

Kernstine et al. [13] investigated the influence of selfactivated movable flaps on airfoil aerodynamic characteristics, including flap size, chord placement, configuration, material selection, and variations in Reynolds number. Johnston and Gopalarathnam [14] designed a lift-enhancing effector device, which was arranged on the upper surface of the airfoil at different angles. The experimental results show that the device can increase the lift coefficient and stall attack angle. Allemand and Altman [15] placed the flap on three different camber airfoils of NACA0012, USA-28, and Eppler-423. The experimental results showed that the flap can improve the stall characteristics. Liu et al. [16] placed the flap on NACA0012 airfoil trailing edge, which substantially extended the airfoil trailing edge. The experimental results showed that the lift coefficient significantly increased. Ge et al. [17] extracted the bionic airfoil from the wing of the long-eared owl and cut out a slat on the upper surface of the 


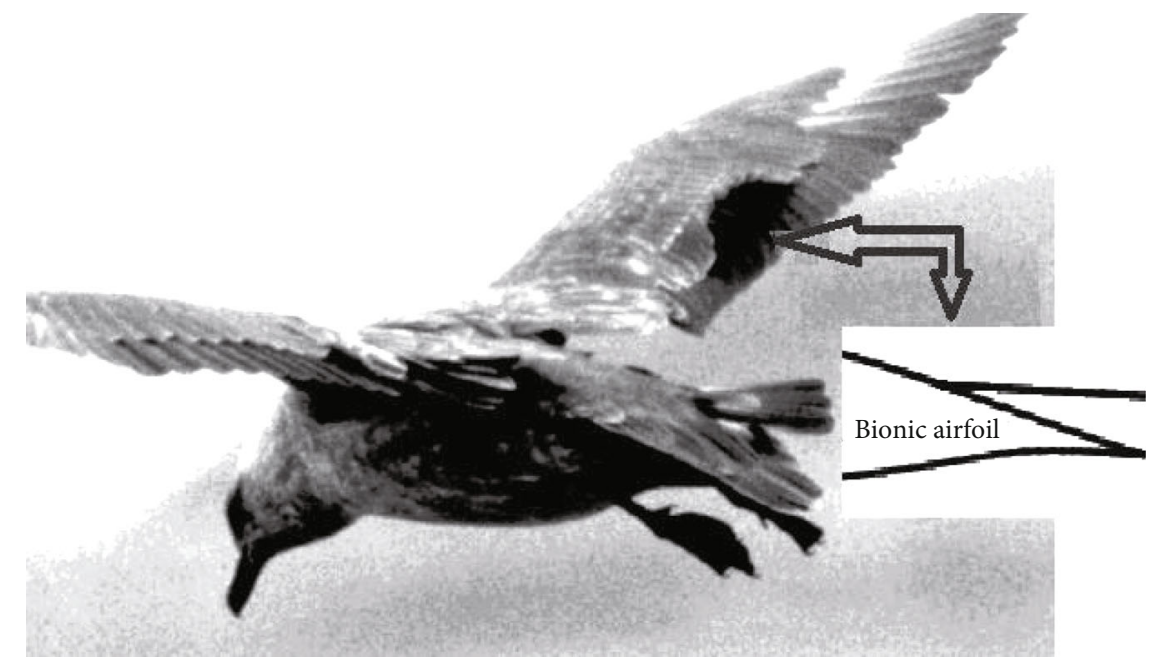

FIGURE 1: A bird's wing with popped up feathers to prevent further proliferation of flow separation [2].

bionic airfoil leading edge to form a multielement airfoil and then carried out the numerical simulation. Rosti et al. [18] focused on the use of passive, self-actuated flaps as lift enhancement devices in nominally stalled conditions. They firstly designed the optimal motion parameter configuration for flap in 2D case and then focused on the interaction between the self-actuated flap devices and the unsteady flow field generated by wing at high angle of attack and how to improve the aerodynamic efficiency of a stalled wing. Meyer et al. [10, 19] conducted a numerical simulation of static flap applied to HQ17 airfoil and focused on the influence of flap deflection angle on airfoil aerodynamic characteristics. Based on this, the study on the influence of flow around the HQ17 airfoil with freely movable flap was conducted. Finally, some progress in the flight test of flap was described. Jost et al. [20] studied the three-dimensional unsteady effect of the trailing edge flap. Beaudoin and Aider [21] studied the effect of small flaps on the lift and drag on a threedimensional blunt body by experimental method. Castaignet et al. [22] tested the configuration of wind turbine blade trailing edge with small flaps and found that the blade load can be reduced.

Scholars from various countries have conducted numerous studies on bionic airfoils by wind tunnel tests or numerical simulations. However, there are few studies on the layouts of bionic airfoils, as well as the influence of the key geometric parameters of bionic airfoil on aerodynamic performance. Therefore, the purposes of this study are twofold: to study bionic airfoil layouts and to investigate the influence of the geometric parameters, including the angle, position, and shape, on aerodynamic characteristics of the bionic airfoil. This study reveals the flow details of the bionic airfoil, explores the key geometric parameters effect on airfoil aerodynamic characteristics, finds an optimal bionic flap shape, and provides theoretical and data support for bionic airfoil application in aeronautical engineering.

\section{Numerical Method Validation}

In this work, the implicit method was used to solve the steady Reynolds-Averaged Navier-Stokes (RANS) equations, and the second-order upwind scheme was used for spatial discretization. The SST transition model was adopted to calculate the viscosity. The boundary conditions were the nonslip wall boundary condition and the far-field boundary condition based on the characteristics of the flow.

In the mesh strategy, C-mesh was calculated. And the normal layers were 120 , the wrap-around airfoil points were 280 , and the cut points were 60 . The first layer spacing was $1 \times 10^{-5} \mathrm{c}$, and total number of cells was $4.8 \times 10^{4}$.

To verify the CFD method in this work, the calculated results were compared with other researches. The calculated model was HQ17 airfoil. The calculation conditions were $\mathrm{Re}=1,000,000$ and $\mathrm{Ma}=0.1$.

For the clean airfoil, the simulation results were compared with the experimental data in article [12] and the calculated data in article [19]. The calculated mesh and result are shown in Figure 2. The $y+$ value is about 1.0. It can be seen that the lift coefficient was nonlinear after AoA $=7^{\circ}$, and the stall AoA was $15^{\circ}$. The lift curve showed that the stall flow occurring at the trailing edge of the airfoil developed slowly toward the leading edge. These simulation results were in good agreement with the experimental data in article [12].

For the controlled airfoil with a trailing edge flap, the simulation results were compared with the result in article [19]. The flap with a length of $20 \% c$ was located at $80 \% c$, and the angle between flap and airfoil upper surface was $14^{\circ}$. The calculated mesh and result are shown in Figure 3. It can be seen that the numerical results of this paper were consistent with those of Meyer et al. [19], and there was a small difference at large AoAs.

The above results showed that the CFD method in this work was effective for both the clean airfoil and controlled airfoil with a trailing edge flap. 

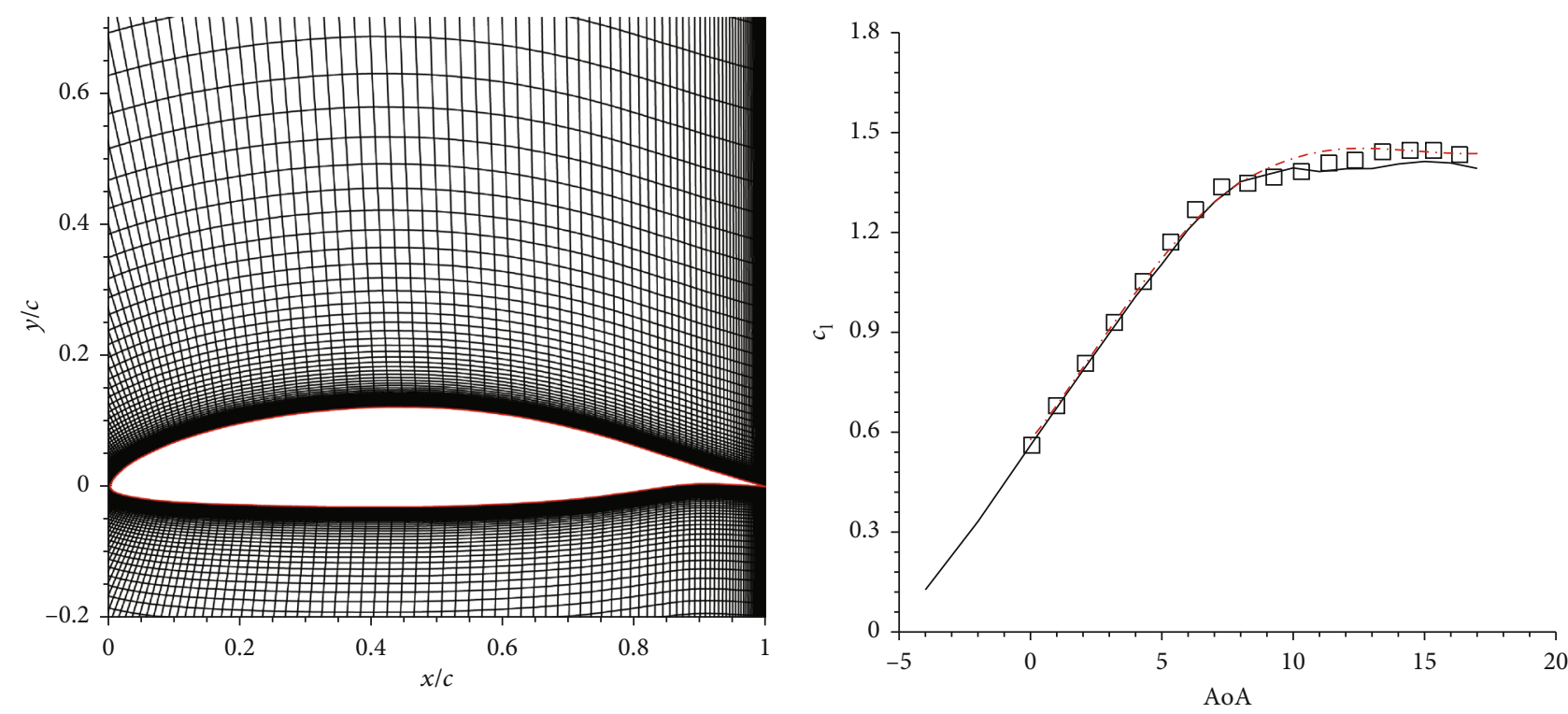

— CFD_In this paper
-. CFD_Meye ret al [19]
$\square \quad$ Exp. data [12]

(a)

(b)

Figure 2: Clean airfoil.

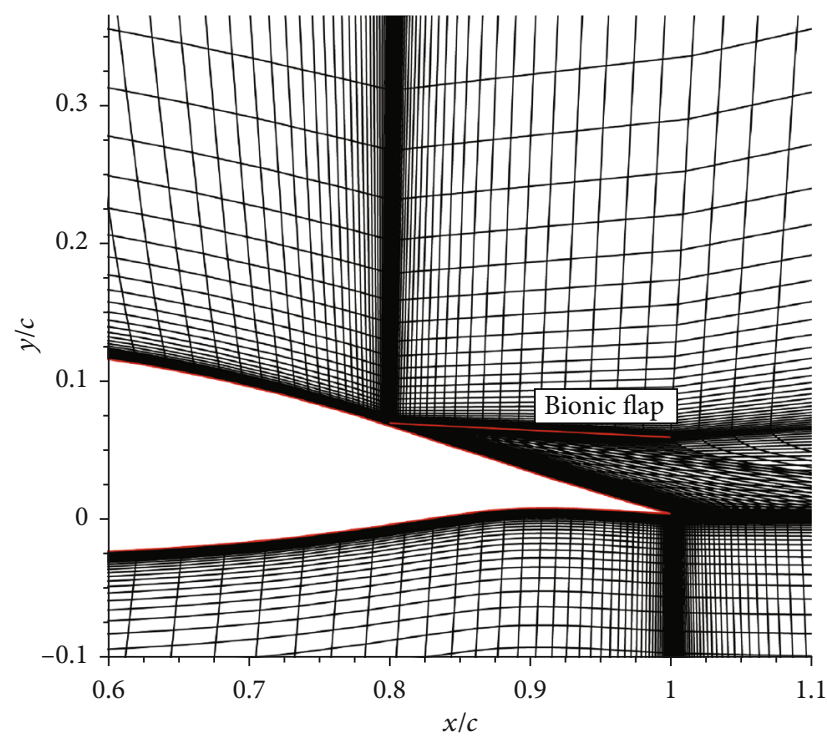

(a)

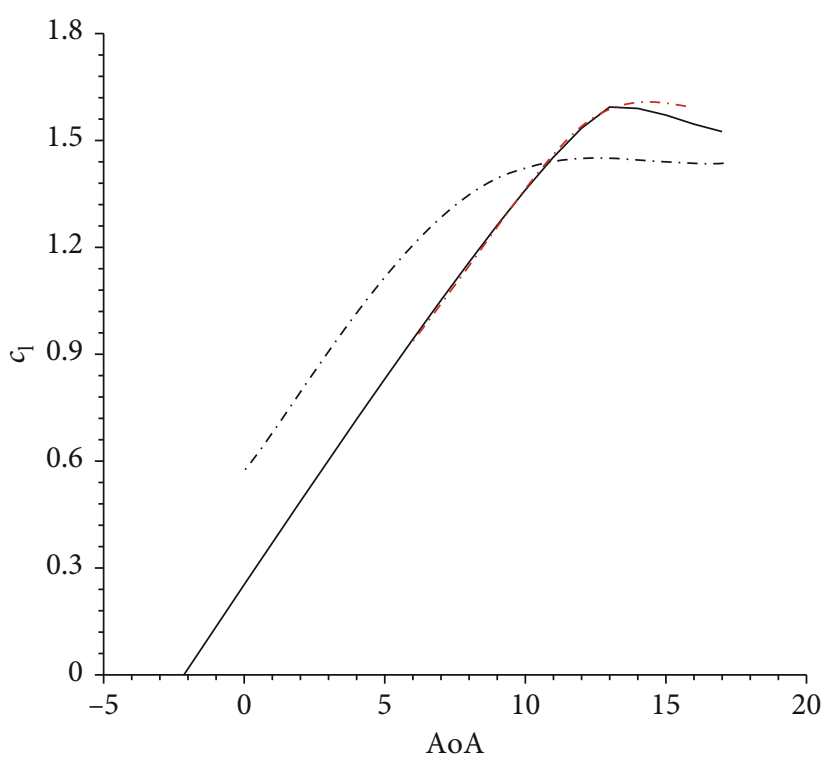

... Baseline_Meyer et al [19]

... - Flap_Meyer et al [19]

_ Flap_In this paper

Figure 3: Airfoil with flow control by trailing edge flap. 


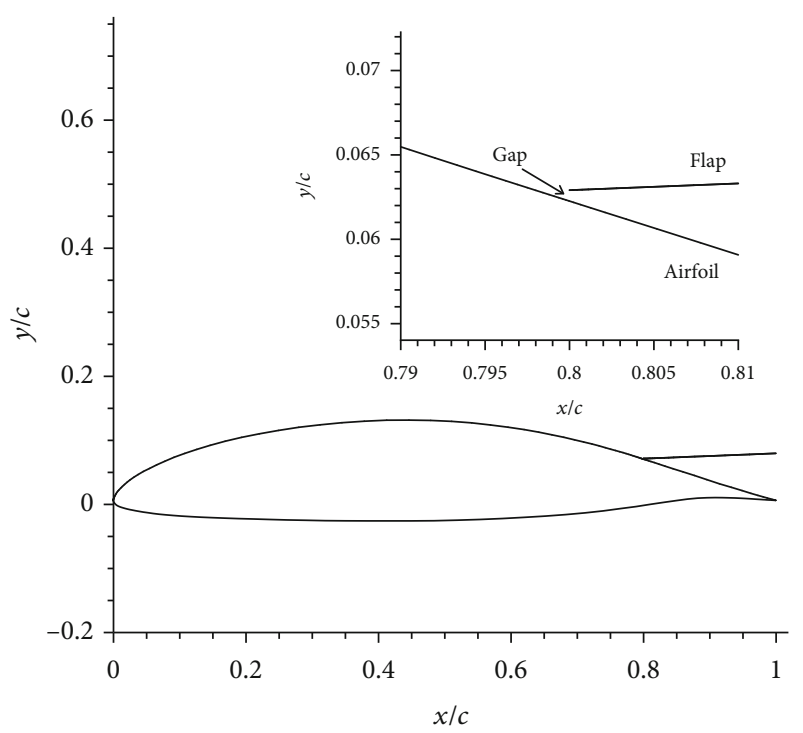

(a)

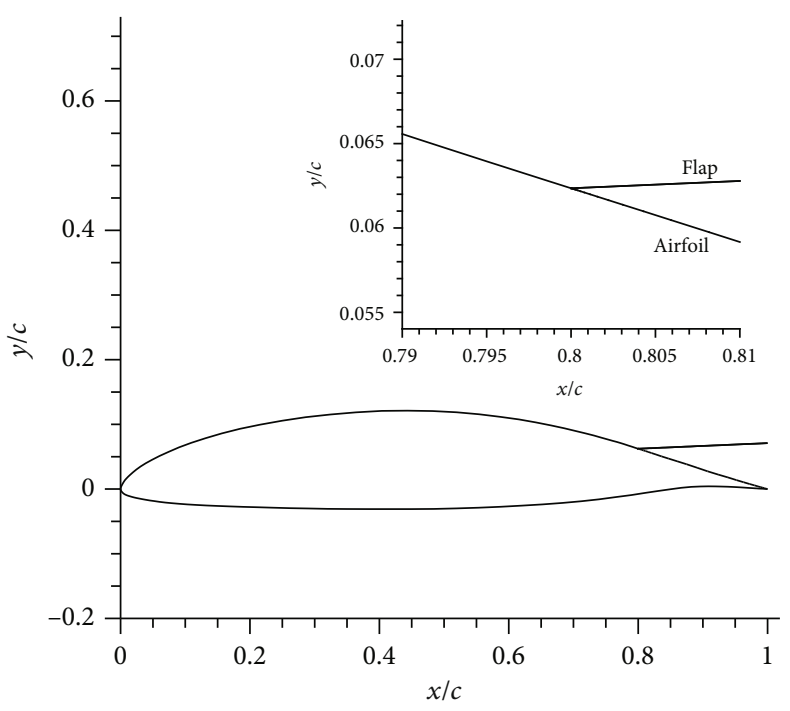

(b)

FIgURE 4: Schematic of bionic airfoil layouts.

TABle 1: Parameters in different configurations.

\begin{tabular}{lccccc}
\hline Layout & Case & $\begin{array}{c}\text { Flap } \\
\text { gap }\end{array}$ & $\begin{array}{c}\text { Flap } \\
\text { angle }\end{array}$ & $\begin{array}{c}\text { Flap } \\
\text { position }\end{array}$ & Flap shape \\
\hline & 1 & $0.0006 c$ & $-5.71^{\circ}$ & $80 \% c$ & Straight line \\
& 2 & $0.0006 c$ & $-2.86^{\circ}$ & $80 \% c$ & Straight line \\
& 3 & $0.0006 c$ & $2.29^{\circ}$ & $80 \% c$ & Straight line \\
& 4 & $0.0006 c$ & $7.41^{\circ}$ & $80 \% c$ & Straight line \\
Layout & 5 & $0.0006 c$ & $12.41^{\circ}$ & $80 \% c$ & Straight line \\
& 6 & $0.0006 c$ & $-2.86^{\circ}$ & $80 \% c$ & Sine $\left(\varphi=0^{\circ}\right)$ \\
& 7 & $0.0006 c$ & $-2.86^{\circ}$ & $80 \% c$ & Sine $\left(\varphi=180^{\circ}\right.$ \\
& 8 & $0.001 c$ & $-2.86^{\circ}$ & $80 \% c$ & Straight line \\
& 9 & $0.0014 c$ & $-2.86^{\circ}$ & $80 \% c$ & Straight line \\
\hline \multirow{2}{*}{ Layout } & 1 & 0 & $-2.68^{\circ}$ & $80 \% c$ & Straight line \\
& 2 & 0 & $-0.1^{\circ}$ & $80 \% c$ & Straight line \\
& 4 & 0 & $2.48^{\circ}$ & $80 \% c$ & Straight line \\
& 5 & 0 & $-2.68^{\circ}$ & $80 \% c$ & Sine $\left(\varphi=0^{\circ}\right)$ \\
& 6 & 0 & $-2.68^{\circ}$ & $80 \% c$ & Sine $\left(\varphi=180^{\circ}\right.$ \\
& & & $85 \% c$ & Straight line \\
\hline
\end{tabular}

\section{Bionic Airfoil Layout}

The bionic airfoil examined in this study consisted of an HQ17 airfoil and a flap of $20 \% c$. Two kinds of bionic airfoil layouts were investigated. The first one was that there was a tiny gap between the airfoil and the small flap, and the second one had no gap, as shown in Figure 4. Here, the gap was defined as the minimum distance between the airfoil upper surface and flap.

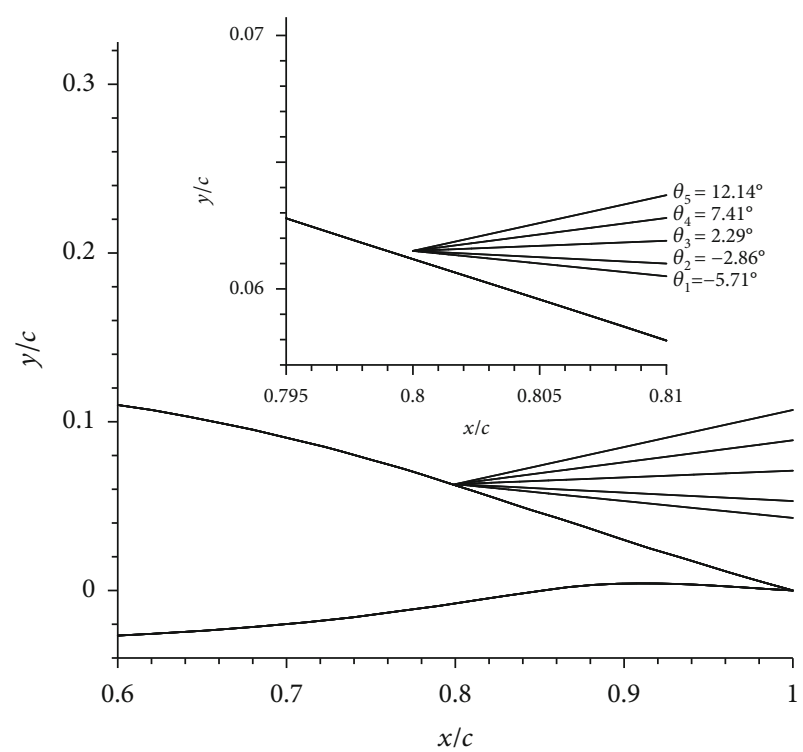

FIGURE 5: Schematic of the bionic airfoil with different values of $\theta$.

To investigate the effect of the flap angle, shape, and position on the aerodynamic characteristics of the airfoil, several configurations were calculated. The parameters of these configurations are in shown Table 1.

In the sinusoidal shape, the amplitude was $0.004 c$, the wavelength was $0.2 c$.

\section{Results and Discussion}

4.1. Layout with a Tiny Gap. Here, the flap angle $\theta$ was defined as the angle between the small flap and the chord line, which can be seen in Figure 5. The length of the flap was $0.2 c$. The fixed end point of the flap was located at the 

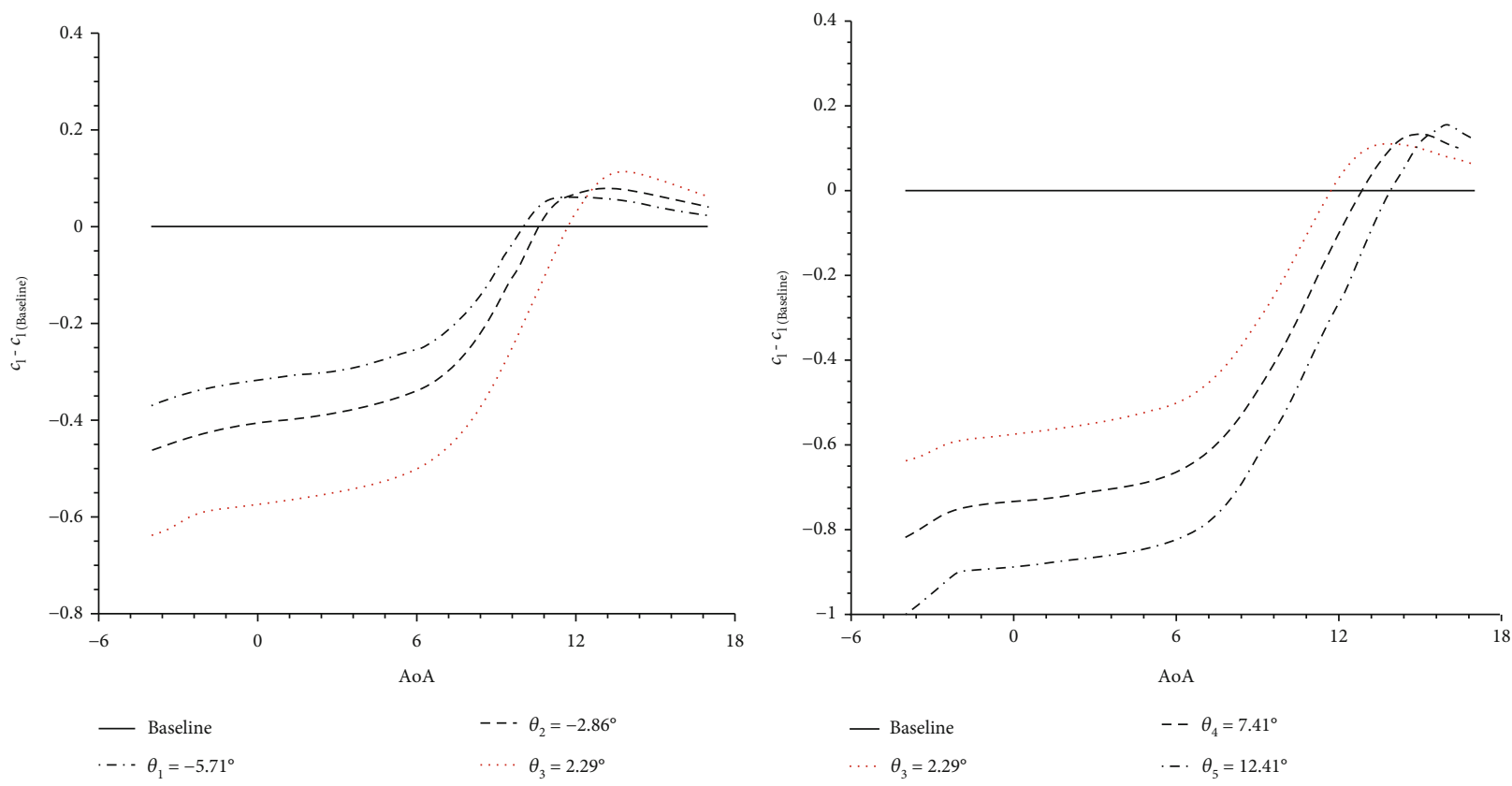

Figure 6: Variations of the lift coefficient curves for the airfoil with different $\theta$.
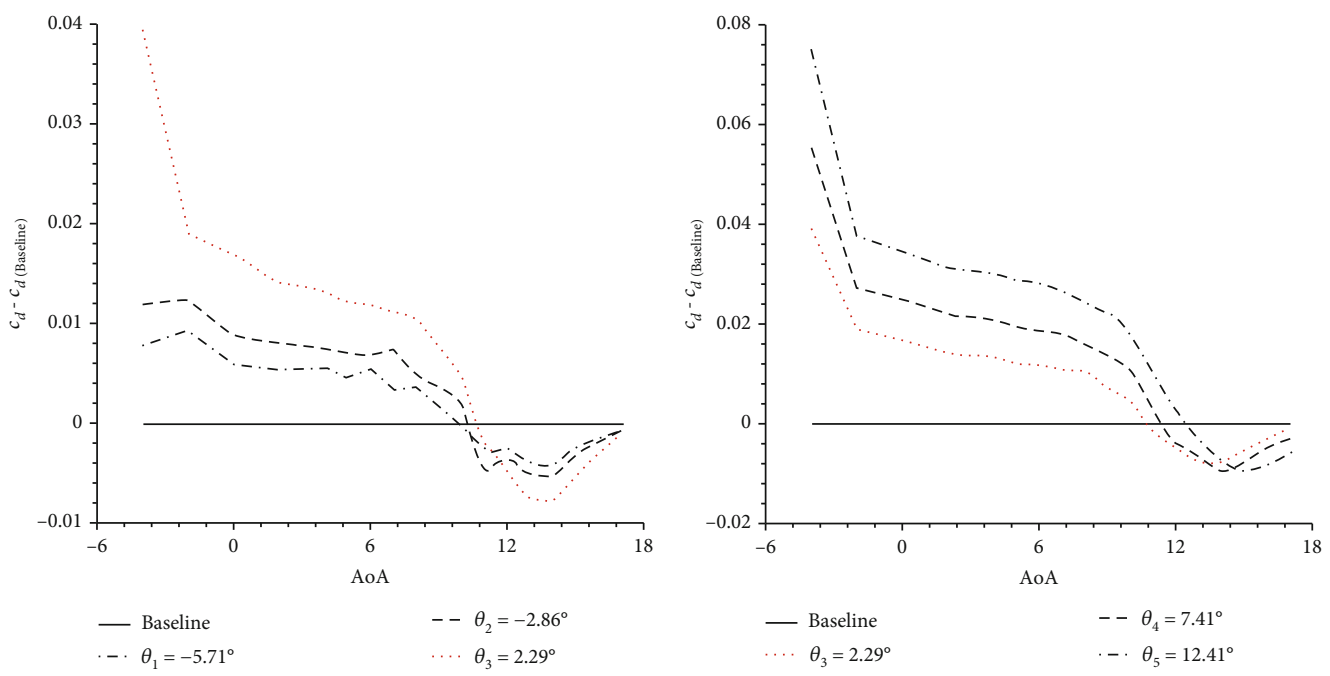

FIGURE 7: Variations of the drag coefficient curves for the airfoil with $\theta$.

position of $80 \% c$, and its normal distance from the airfoil surface was $0.06 \% c$.

Figures 6 and 7 show variations of the lift and drag coefficient curves for the airfoil with different $\theta$. The aerodynamic characteristic curve of the airfoil without the small flap is defined as the baseline airfoil. The figures show that the lift coefficient is greatly reduced in the attention with a small AoA, and the drag is obviously increased. However, at a large angle of attack, the lift coefficient is significantly improved, and the drag is reduced slightly. The maximum lift coefficients of case 1 with $\theta=-5.71^{\circ}, \theta=-2.86^{\circ}, \theta=$
TABLE 2: Effect of the flap angle on the lift-to-drag ratio.

\begin{tabular}{lccccc}
\hline AoA & $\theta=-5.71^{\circ}$ & $\theta=-2.86^{\circ}$ & $\theta=2.29^{\circ}$ & $\theta=7.41^{\circ}$ & $\theta=12.41^{\circ}$ \\
\hline 11 & 6.85 & 9.34 & 0.05 & -9.92 & -19.28 \\
12 & 3.66 & 5.22 & 5.49 & 0.52 & -8.43 \\
13 & 3.70 & 5.14 & 7.23 & 4.62 & -0.37 \\
14 & 2.75 & 3.76 & 5.81 & 6.78 & 3.45 \\
15 & 1.37 & 2.04 & 3.33 & 5.08 & 5.43 \\
16 & 0.80 & 1.09 & 1.75 & 2.66 & 4.45 \\
17 & 0.39 & 0.54 & 0.85 & 1.47 & 2.38 \\
\hline
\end{tabular}



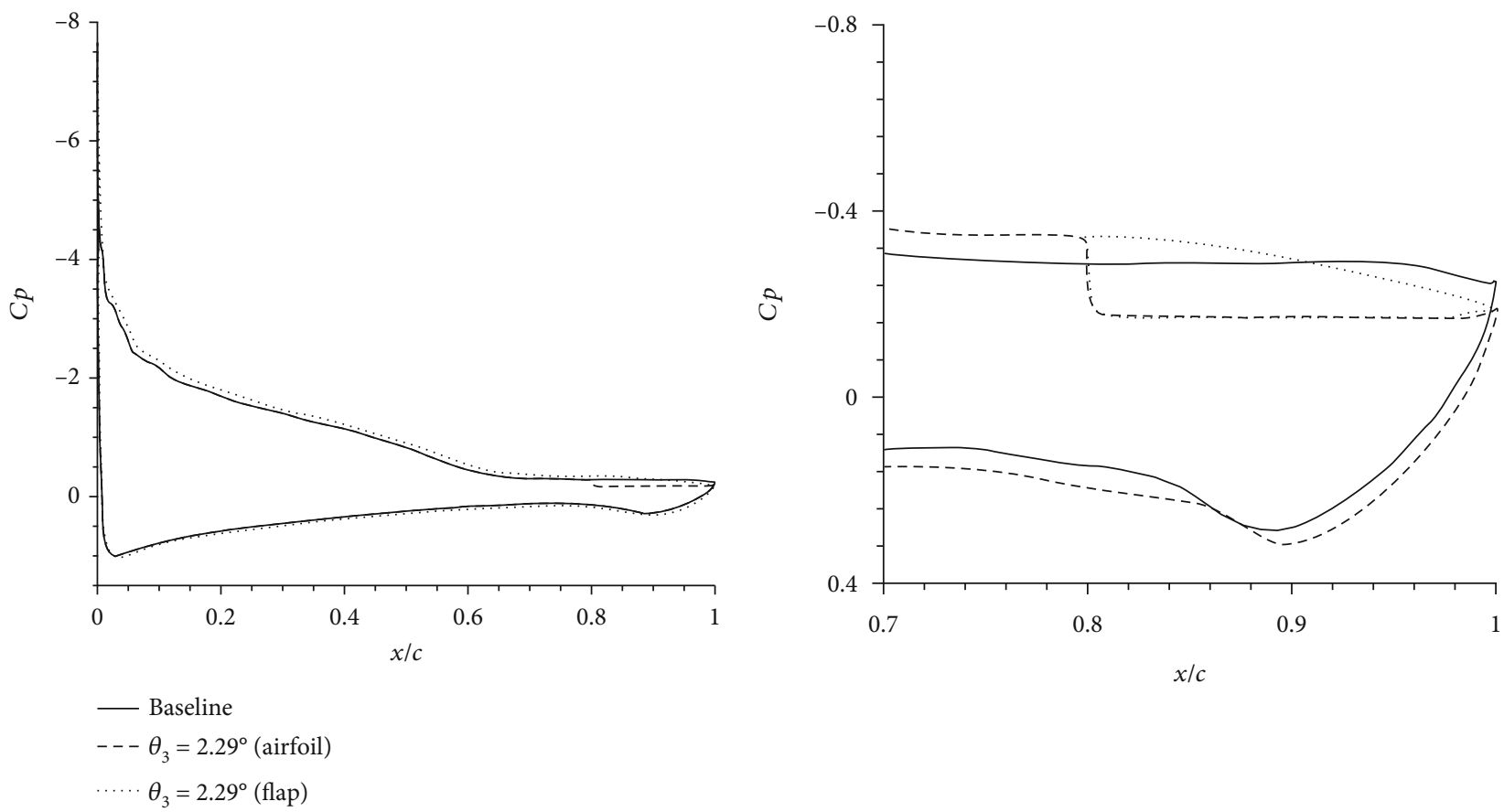

Figure 8: Comparison of pressure distributions of the baseline airfoil and bionic airfoil $\left(\mathrm{AoA}=14^{\circ}\right)$.

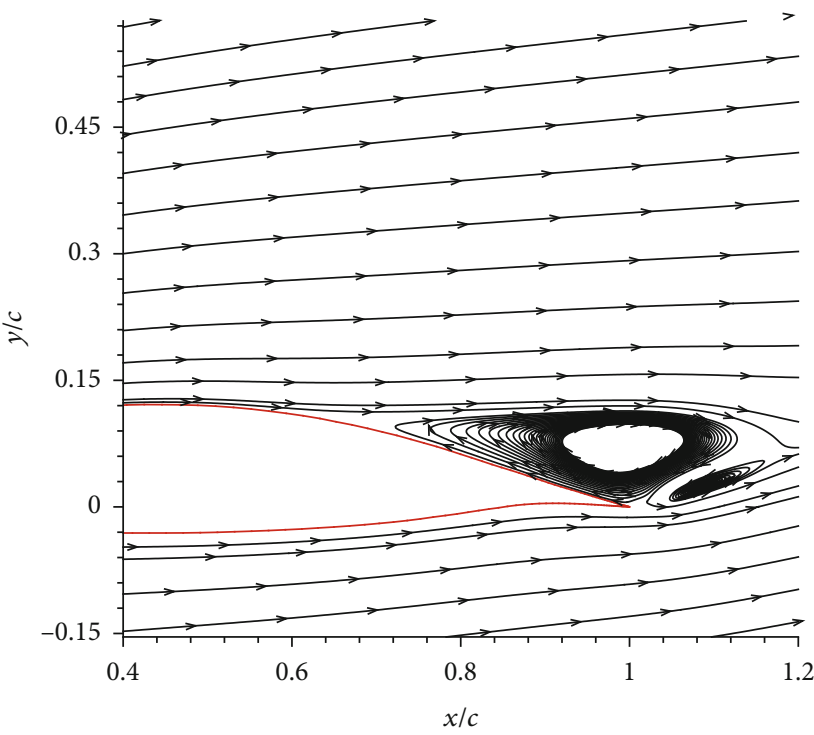

(a) Baseline airfoil

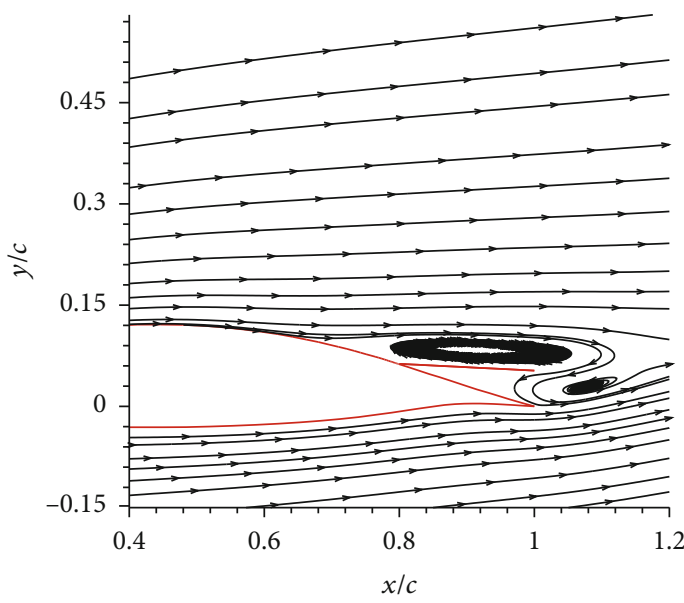

(b) Bionic airfoil $\left(\theta=-2.86^{\circ}\right)$

Figure 9: Comparison of streamlines of the baseline airfoil and bionic airfoil $\left(\mathrm{AoA}=14^{\circ}\right)$.

$2.29^{\circ}, \theta=7.41^{\circ}$, and $\theta=12.41^{\circ}$ are increased by $3.1 \%, 4.9 \%$, $7.5 \%, 9.6 \%$, and $10.9 \%$, respectively. Meanwhile, the drag coefficients are decreased by $7.1 \%, 9.0 \%, 13.3 \%, 11.9 \%$, and $11.0 \%$, respectively. As the flap angle increases, the lift decreases significantly at a small AoA, as the lift increases at a large AoA, the stall AoA is also increased, which is consistent with Meyer et al.'s conclusion [19]. The excessive flap angle (such as $\theta_{5}=12.41^{\circ}$ ) can further increase the maxi- mum lift coefficient, but obviously, the initial AoA to achieve lift-enhancement effect is larger, and the AoA range of lift enhancement is smaller. Therefore, the choice of the flap angle should be compromised between the impact on the lift and the impact on the drag; the flap angle is a critical parameter for the bionic airfoil.

In order to study the effect of the flap angle on the lift-todrag ratio of the HQ17 airfoil, the percentage increase in the 


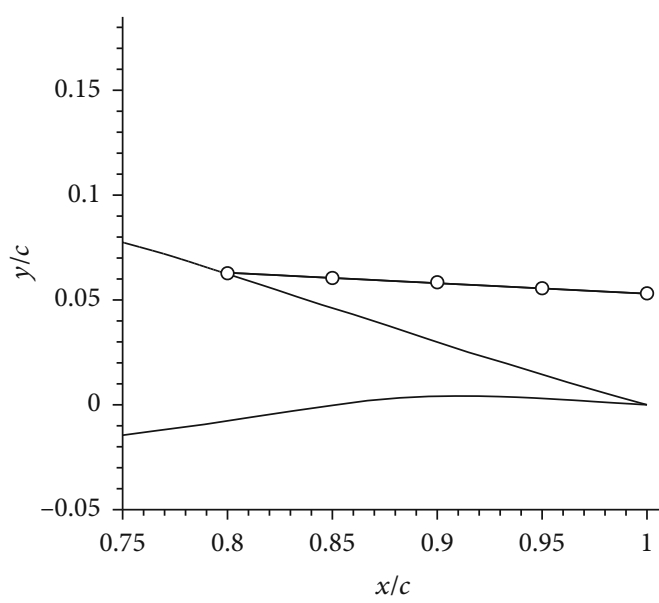

○ Control point

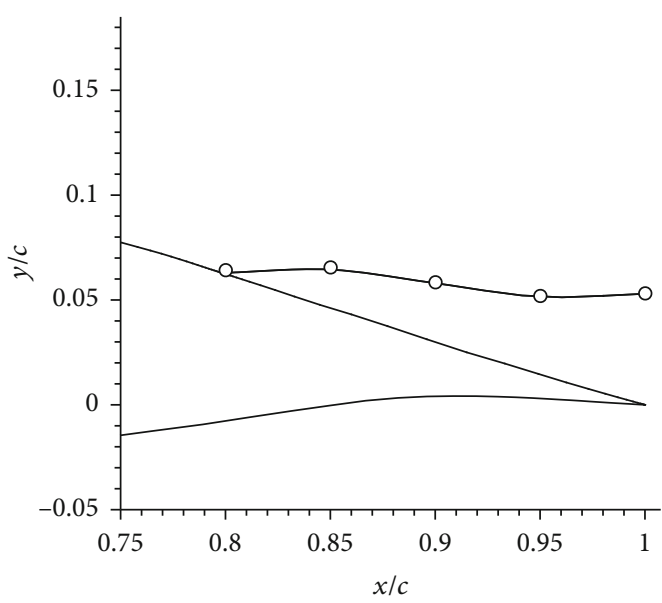

○ Control point

(b) $\mathrm{S} 1$

(a) S0

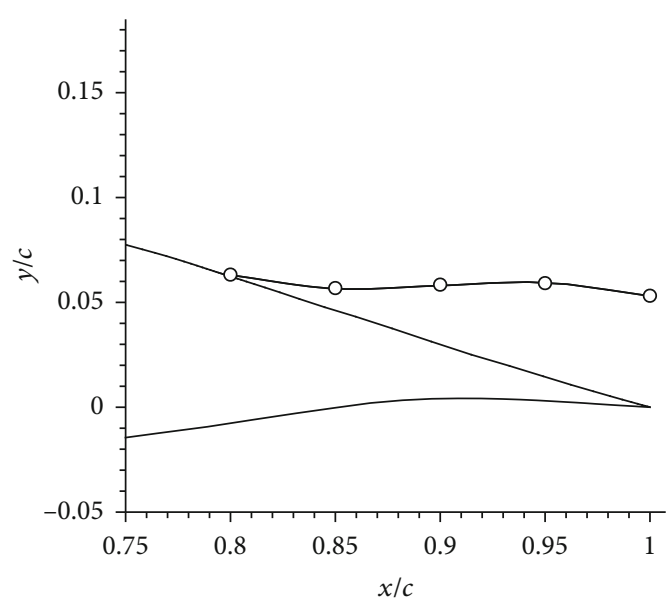

○ Control point

(c) S2

Figure 10: Schematic of the bionic airfoil with three kinds of flap shapes.

lift-to-drag ratio is defined here, i.e., $\left(K_{\theta}-K_{\text {Baseline }}\right) / K_{\text {Baseline}}$, where " $K_{\theta}$ " and " $K_{\text {Baseline, "respectively, represent the lift-to- }}$ drag ratio of the airfoil with and without a flap. The effect of flap angle on the lift-to-drag ratio for the HQ17 airfoil is shown in Table 2 (Layout 1). The results show that the flaps of different angles can increase the lift-to-drag ratio in the stall section. It can be seen from Figures 6 and 7 that the lift-enhancement effect of the flap is remarkable in the stall section, the drag is decreased, and the lift-to-drag ratio is increased. The flap angle is larger, and the attack of angle range of the lift-to-drag ratio enhancement is smaller. By comparing the data in Table 2, it can be found that the lift-enhancement effect of the flap with $\theta=-2.86^{\circ}$ is better than that of the other flaps, and the flap with $\theta=-2.86^{\circ}$ is the best.

Figure 8 compares the pressure distributions of the baseline airfoil and the bionic airfoil with the first layout, showing that the bionic airfoil accelerates the flow on the upper surface and slows down the flow on the lower surface, which results in an increase in the airfoil lift.

Figure 9 compares the streamlines of the baseline airfoil and the bionic airfoil case $2\left(\theta=-2.86^{\circ}\right)$ in the first layout. From the figure, we can see a large and serious separation vortex at the trailing edge of the baseline airfoil; however, there are also two small separation vortices near the flap at the trailing edge of the bionic airfoil. This means that the bionic airfoil can suppress the development of flow separation.

In the following part, we will discuss the effect of the flap shape on the aerodynamic performance of the bionic airfoil, based on the observation of the airfoil with the flap angle of $\theta=-2.86^{\circ}$. The flaps with the straight line and sinusoid curve are analyzed, which are designated as S0, S1, and S2, respectively. S1 and S2 are approximately formed based on the straight flap with five control points, as shown in Figure 10. 


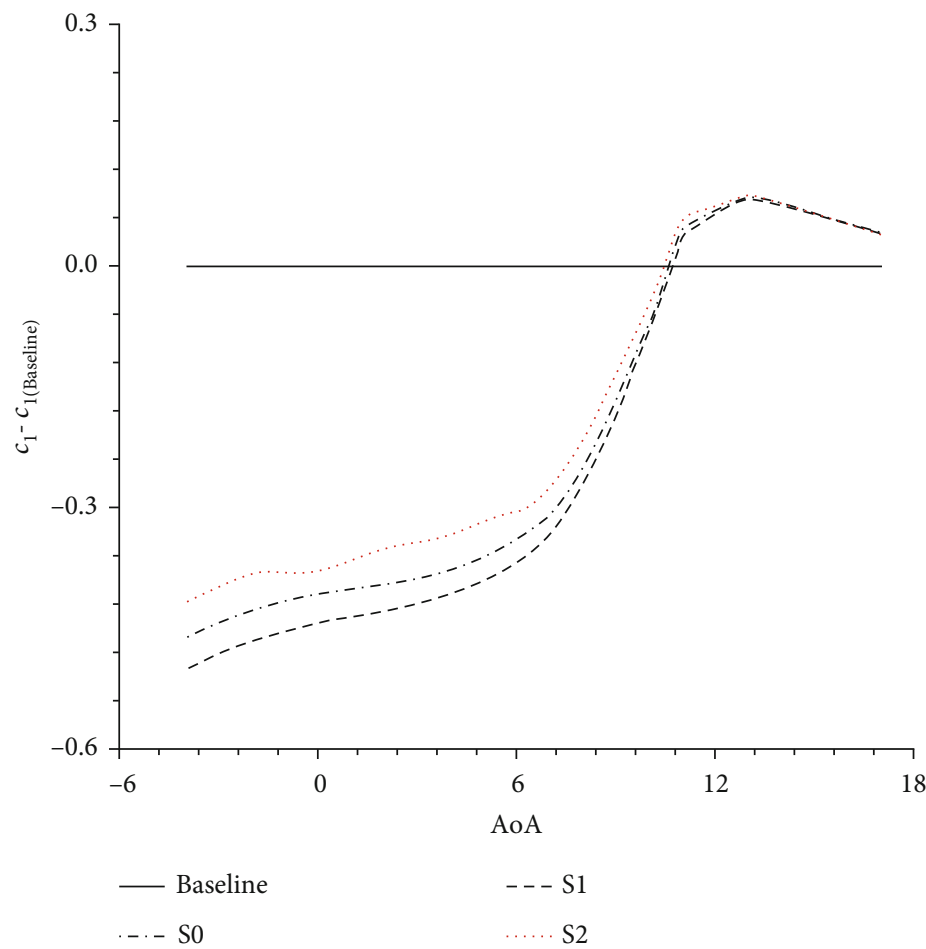

(a) Lift coefficient

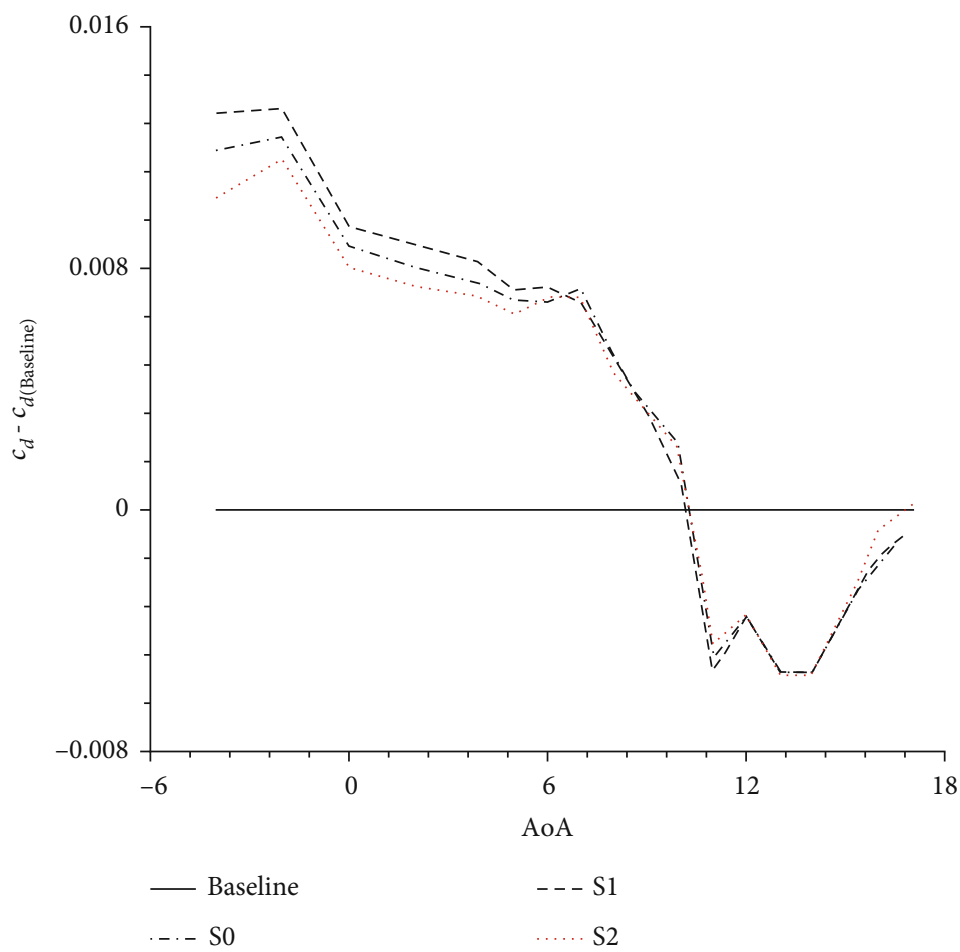

(b) Drag coefficient

FIGURE 11: Variations of the lift and drag coefficients for the airfoil with three flap shapes.

The aerodynamic characteristic curves of the bionic airfoil with three kinds of flap shapes are shown in Figure 11. It can be seen that for all the three flap shapes, the lift coeffi- cient is increased and the drag coefficient is decreased at a large AoA. The maximum lift coefficients of S0, S1, and S2 are increased by $4.9 \%, 4.8 \%$, and $4.9 \%$, respectively. 


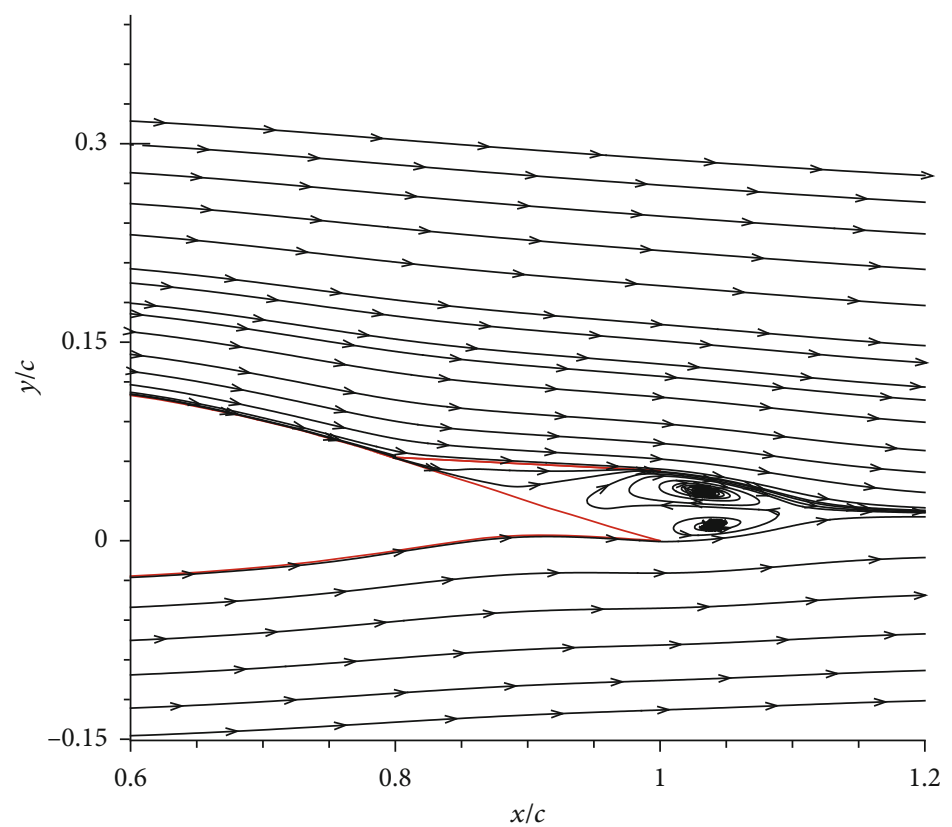

(a) S0

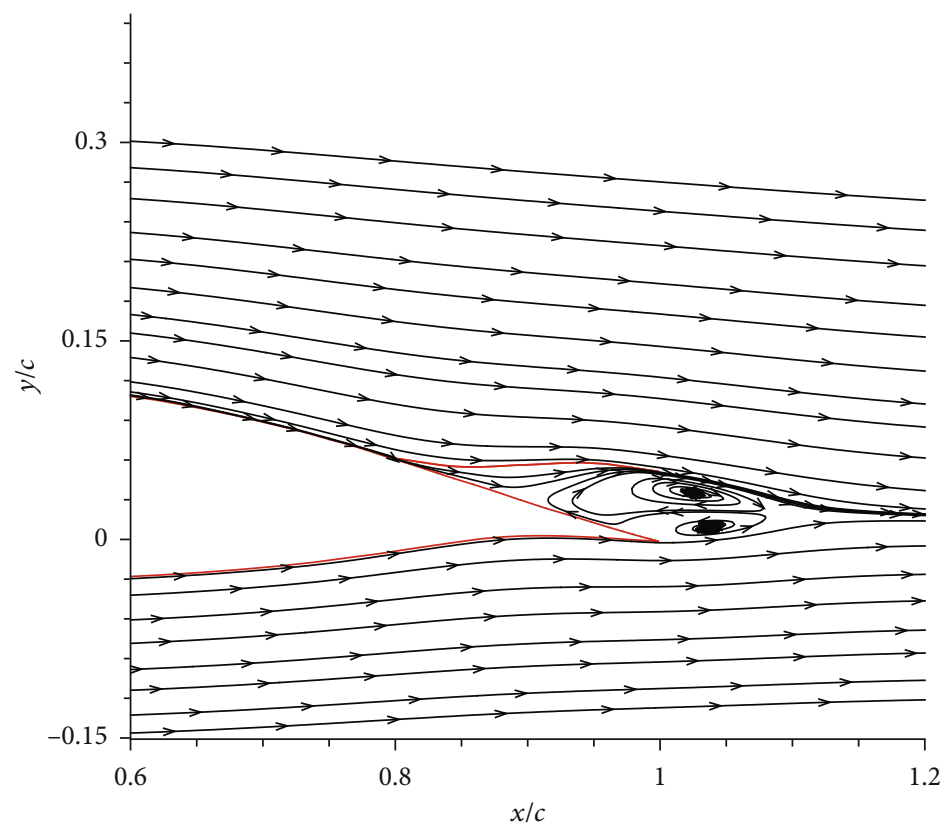

(b) $\mathrm{S} 1$

Figure 12: Continued. 


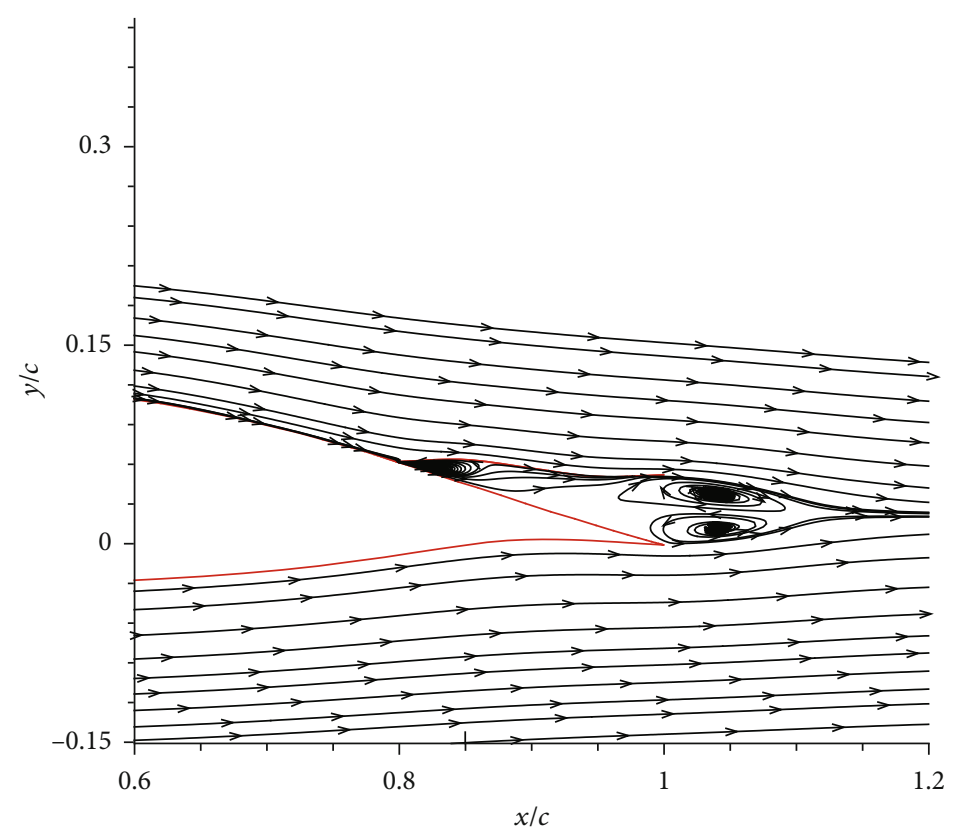

(c) S2

Figure 12: Comparison of streamlines of the airfoil with three flap shapes $\left(\mathrm{AoA}=0^{\circ}\right)$.

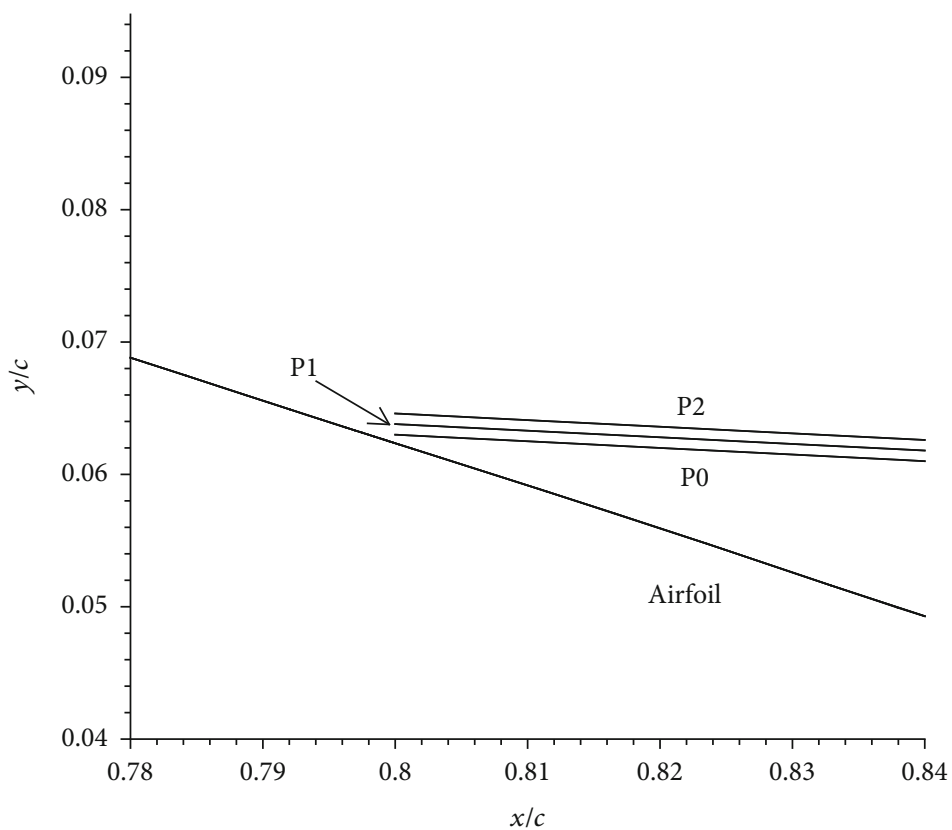

FIGURE 13: Schematic of the bionic airfoil with three flap positions.

Meanwhile, the drag coefficients are decreased by $9.0 \%$, $9.1 \%$, and $9.2 \%$, respectively. However, there is still obvious difference in the aerodynamic characteristic curves at a small AoA. With the respect to the lift and drag, S2 is the best shape.

Figure 12 shows the streamlines of the three configurations S1, S2, and S3 at AoA $=0^{\circ}$. From the perspective of the separation vortex, apart from a pair of reverse separation vortices at the end of the small piece, a tiny separation vortex was formed at the corner between the small piece and the airfoil surface in the case of S1. A pair of reverse separation vortices appeared at the ends of the small piece in the case of S2 and S3. It can be seen that the streamlines passing through the upper and lower surfaces of the airfoil have 


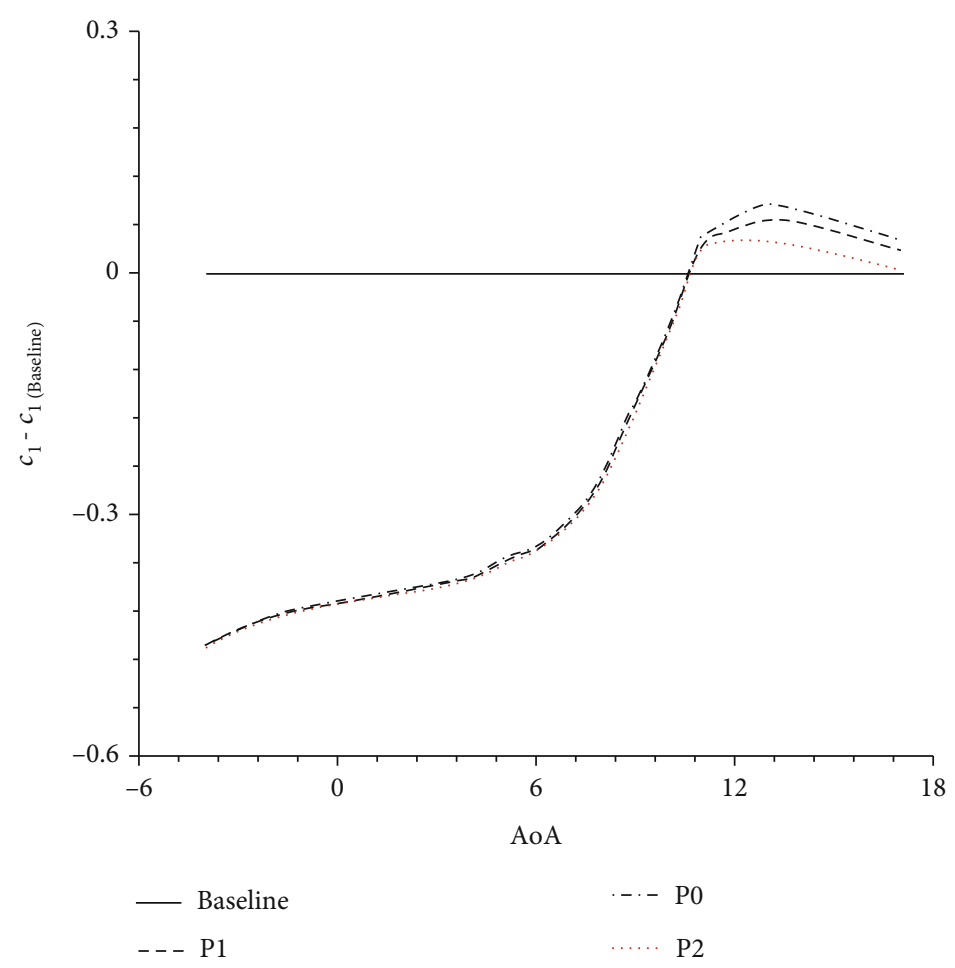

(a) Lift coefficient

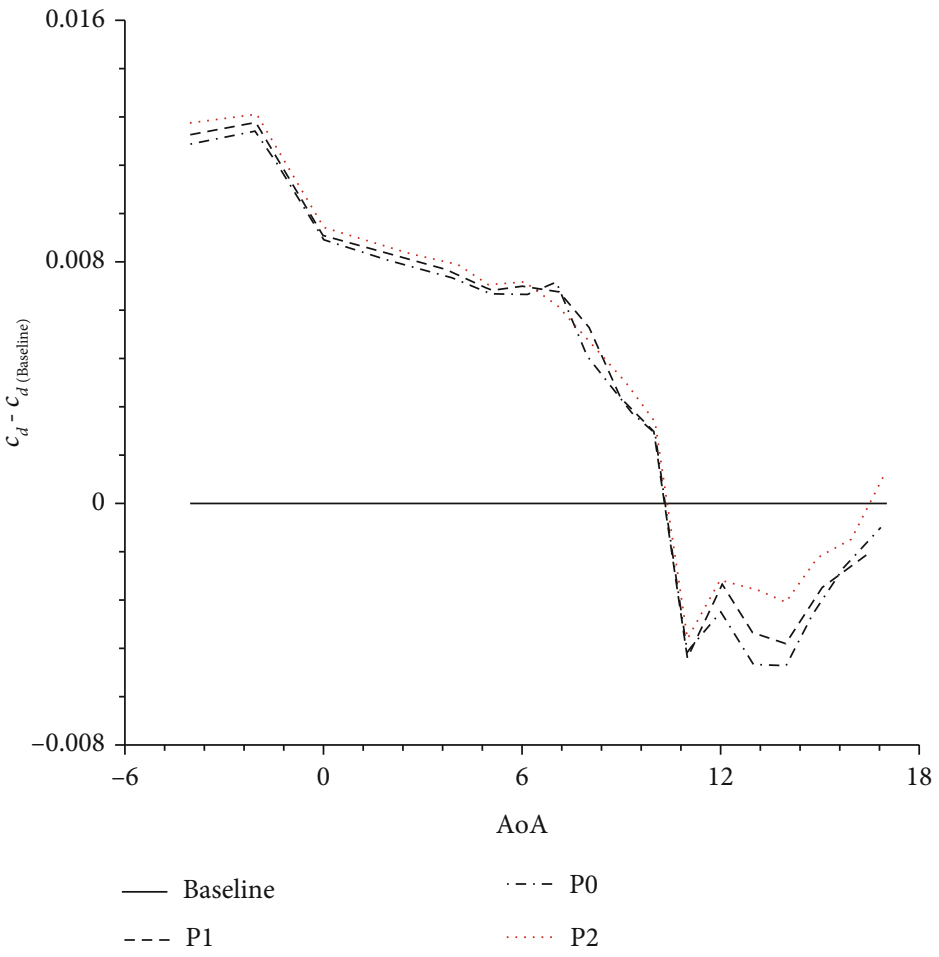

(b) Drag coefficient

Figure 14: Variations of the lift and drag coefficients for the airfoil with three flap positions.

slight differences in the location where they converge near the trailing edge, and the height relationship between the location of the confluence point is $\mathrm{S} 2<\mathrm{S} 0<\mathrm{S} 1$.
Then, this study examines the effect of the flap position (gap height) on the aerodynamic performance of the bionic airfoil. Three positions for the small flap are studied at the 
TABLE 3: Effect of the flap positions and shapes on the lift-to-drag ratio.

\begin{tabular}{lccccc}
\hline AoA $\left(^{\circ}\right)$ & P0 $($ S0 $)$ & P1 & P2 & S1 & S2 \\
\hline 11 & 9.34 & 9.64 & 8.11 & 9.12 & 9.07 \\
12 & 5.22 & 3.76 & 3.36 & 5.09 & 5.23 \\
13 & 5.14 & 3.97 & 2.48 & 5.12 & 5.33 \\
14 & 3.76 & 3.09 & 1.96 & 3.76 & 3.79 \\
15 & 2.04 & 1.69 & 0.90 & 2.03 & 2.01 \\
16 & 1.09 & 0.97 & 0.45 & 1.02 & 0.81 \\
17 & 0.54 & 0.48 & 0.14 & 0.55 & 0.40 \\
\hline
\end{tabular}

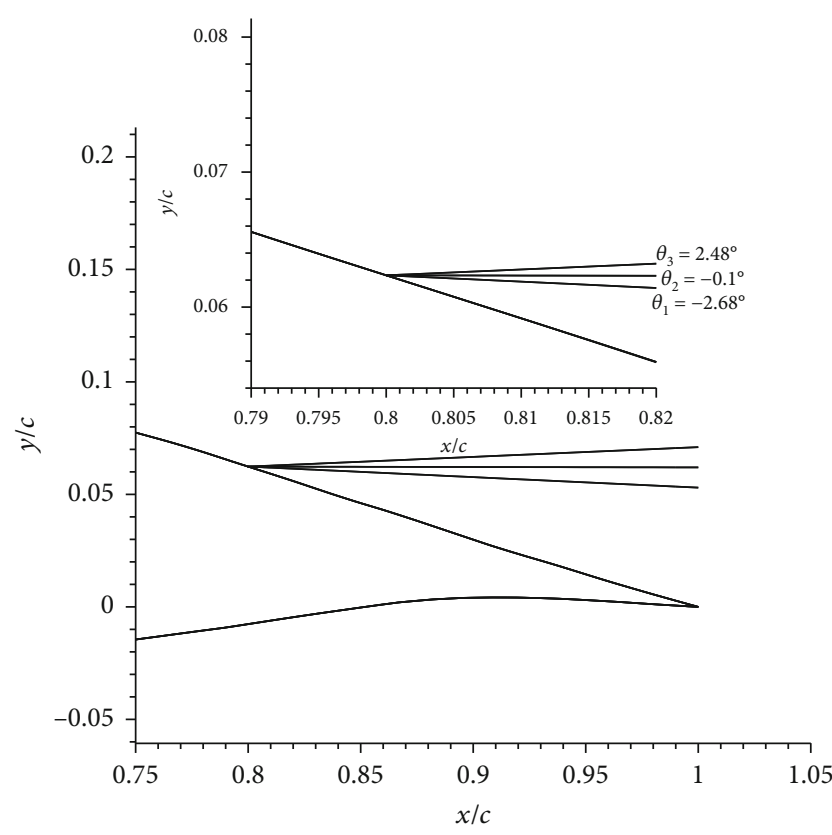

FIGURE 15: Schematic of the bionic airfoil with different $\theta$.

flap angle of $\theta_{2}=-2.86^{\circ}$, as shown in Figure 13. Based on the original flap position (P0), the flap is shifted along the $y$ -coordinate by $0.08 \% c$ and $0.16 \% c$, which are the positions $\mathrm{P} 1$ and $\mathrm{P} 2$, respectively.

The aerodynamic characteristic curves of the bionic airfoil with three flap positions are shown in Figure 14. The figure shows that the lift and drag curves for P0, P1, and P2 almost coincide at a small AoA and that the lift is increased and the drag reduced at a large AoA. With the increase of gap height, the improvement of stall characteristics is weakened, and the increase of lift and the decrease of drag are both reduced. The flap position P0 is the best for improving the stall characteristics, which means that the farther the flap from the airfoil surface, the weaker the improvement in stall characteristics. Therefore, the flap position is a critical parameter for the bionic airfoil.

The effect of flap positions and shapes on the lift-to-drag ratio for the HQ17 airfoil is shown in Table 3. The results show that the flap position $\mathrm{P} 0$ is the best for improving the stall characteristics among the three flap positions, and the three kinds of flap shapes are equally capable of improving stall characteristics. In all the flap positions and shapes, the effective AoA range for improving the lift and lift-to-drag ratio can reach $7^{\circ}$. By comparing the data in Table 3 and Figure 11, it can be found that S2 is the best.

4.2. Layout with No Gap. In this subsection, we will analyze how the angle, shape, and position parameters of the small flap sticking to the airfoil affect the aerodynamic characteristics of the airfoil. The angle definition of the small flap in Layout 2 is the same as that in Layout 1 . The flap in the second airfoil layout has a length of $20 \% c$, with its leading end point fixed and the trailing end point rotated around the fixed end point to change the flap angle. The fixed end point of the flap is located at the position of $80 \% c$. Figure 15 shows the schematic of the flaps with $\theta=-2.68^{\circ},-0.1^{\circ}$, and $2.48^{\circ}$.

Figure 16 shows the lift and drag coefficient curves of the airfoil with different $\theta$. As can be seen in this figure, at a small AoA, the lift coefficient is greatly reduced and the drag is obviously increased; at a large AoA, however, the lift coefficient is significantly improved, and the drag is reduced. As the flap angle increases, the maximum lift coefficient and the stall AoA also increase, and the initial AoA to achieve lift increase is gradually larger. The maximum lift coefficients of case 2 with $\theta=-2.68^{\circ}, \theta=-0.10^{\circ}$, and $\theta=2.48^{\circ}$ are increased by $5.0 \%, 6.2 \%$, and $7.9 \%$, respectively. Meanwhile, the drag coefficients are decreased by $8.9 \%, 10.9 \%$, and $13.6 \%$, respectively.

The following part will discuss the effect of the flap shape on the aerodynamic performance of the bionic airfoil. The shape definition of the small flap in Layout 2 is the same as that in Layout 1 . When $\theta=-2.68^{\circ}$, the flap is of a straight line shape (S0). Based on S0, S1 and S2 are approximately formed with five control points. Figure 17 shows the schematic of the bionic airfoil with three flap shapes.

The aerodynamic characteristic curves of the bionic airfoil with the three flap shapes are shown in Figure 18. It can be seen that, at a small AoAs, the lift coefficient is greatly reduced, but the drag coefficient is obviously increased. At a large AoA, the lift coefficient is significantly improved, but the drag coefficient is reduced. However, there is still a small difference in aerodynamic characteristic curves at a small AoA. With respect to the lift and drag, S2 is the best shape.

The effect of the flap position on the aerodynamic performance of the bionic airfoil is introduced below. When $\theta$ $=-2.68^{\circ}$, the flap is of a straight line shape, named P0. We translate the flap P0 along the negative direction of the $y$ -axis and move the leading end point from the upper surface of the airfoil $80 \% c$ to $85 \% c$ to obtain the flap P1. Figure 19 shows the schematic of the bionic airfoil with two flap positions.

Figure 20 presents the lift and drag coefficient curves of the airfoil with two flap positions. The figure shows that P0 significantly improves the stall characteristics of the airfoil, but P1 shows no improvement, which is not the same as Layout 1. Therefore, the flap position is a critical parameter for the bionic airfoil. 


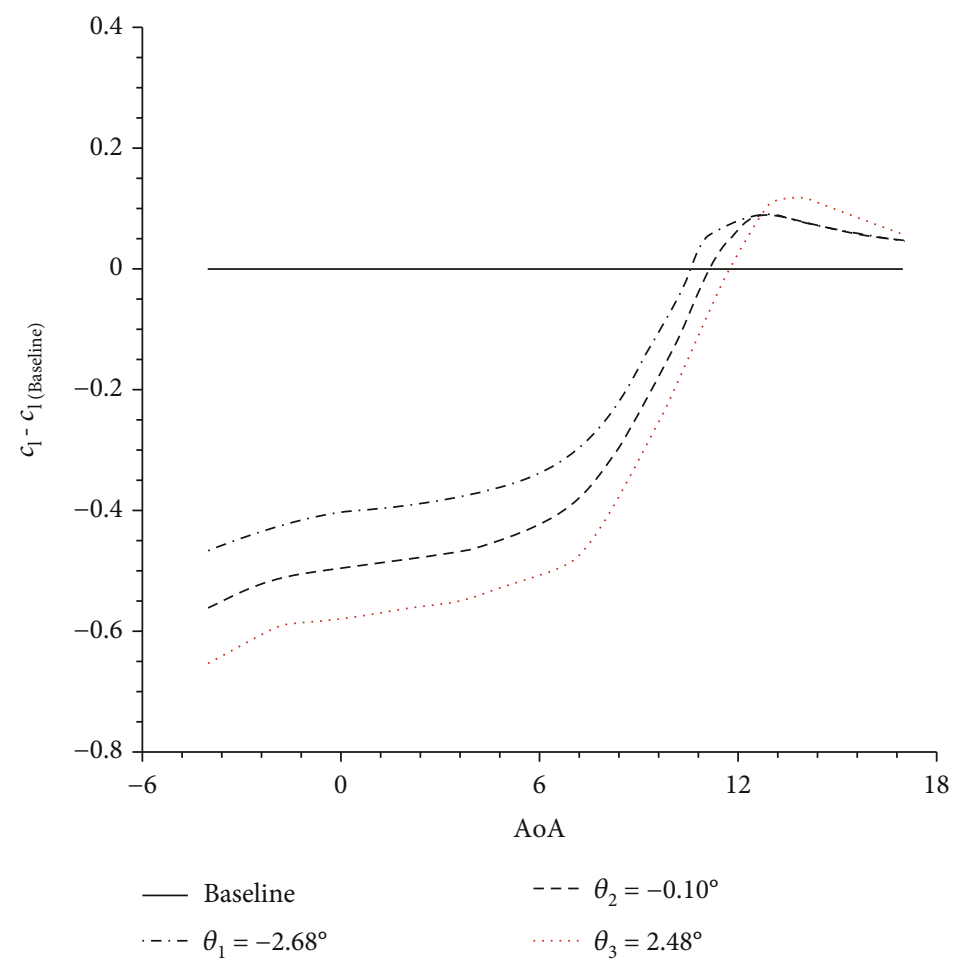

(a) Lift coefficient

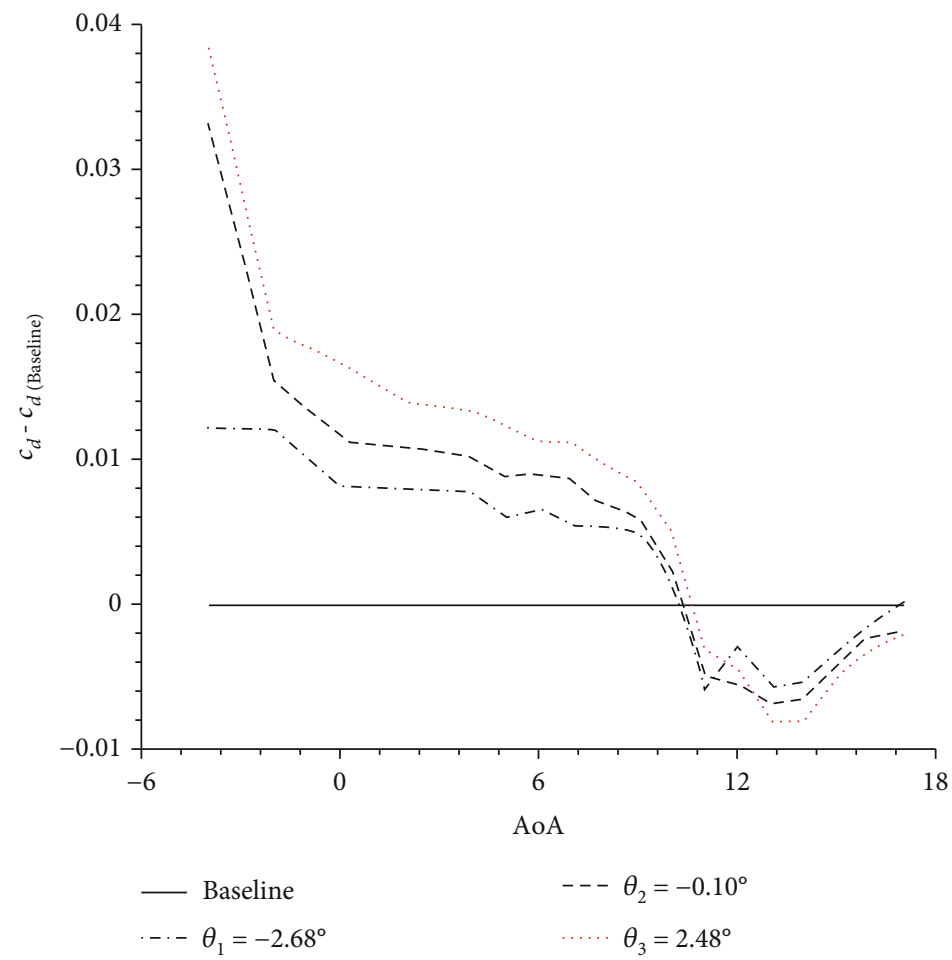

(b) Drag coefficient

FIgure 16: Variations of the lift and drag coefficient curves for the airfoil with different $\theta$.

The effect of flap angles, positions, and shapes on the lift-to-drag ratio for the HQ17 airfoil is shown in Table 4. The results show that the flap shape S2 is the best for improving the stall characteristics among all the flap positions, angles, and shapes, and the effective AoA range for improving the lift-to-drag ratio can reach $7^{\circ}$. By comparing the data in Table 4 and Figure 18, it can be found that $\mathrm{S} 2$ is the best. 


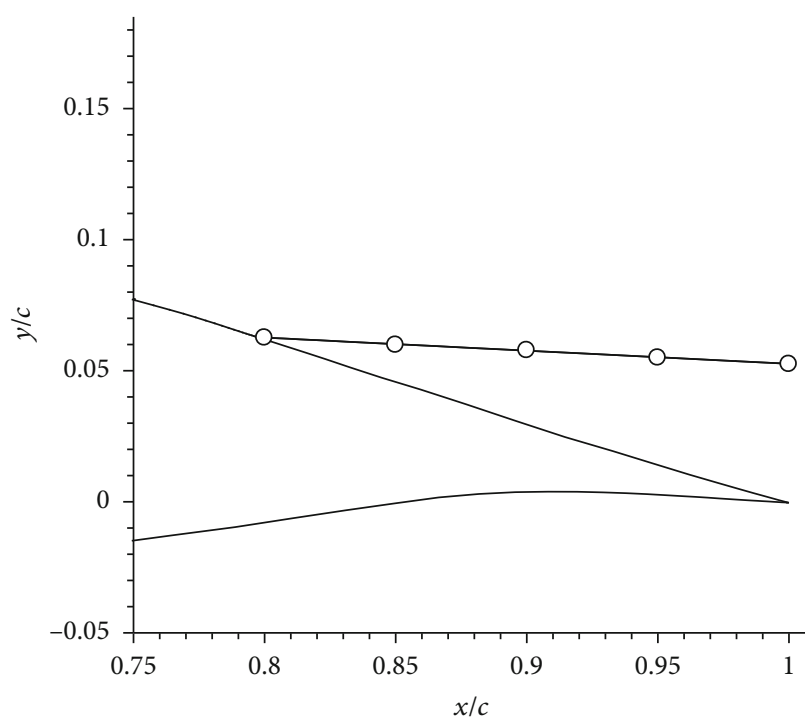

O Control point

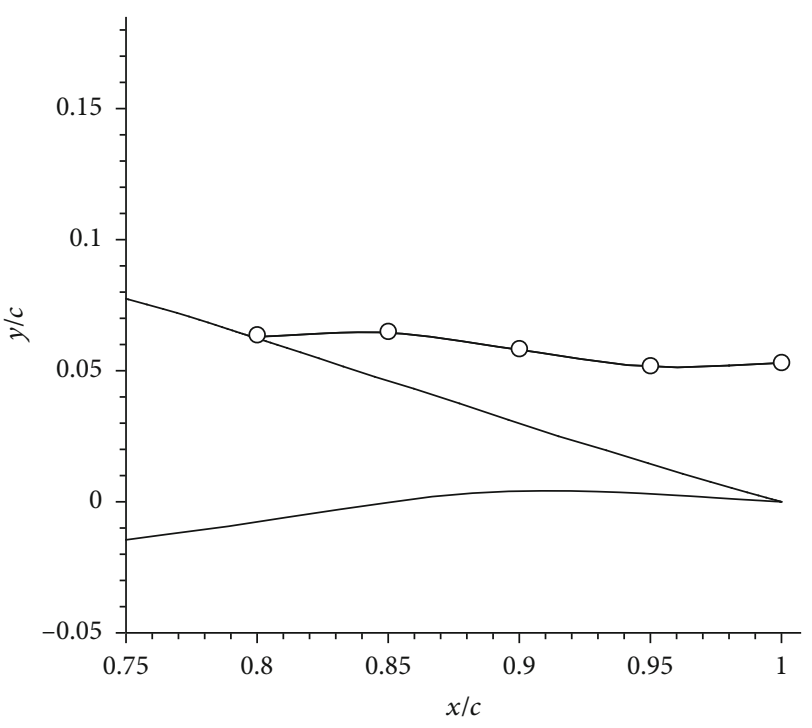

O Control point

(a) S0

(b) S1

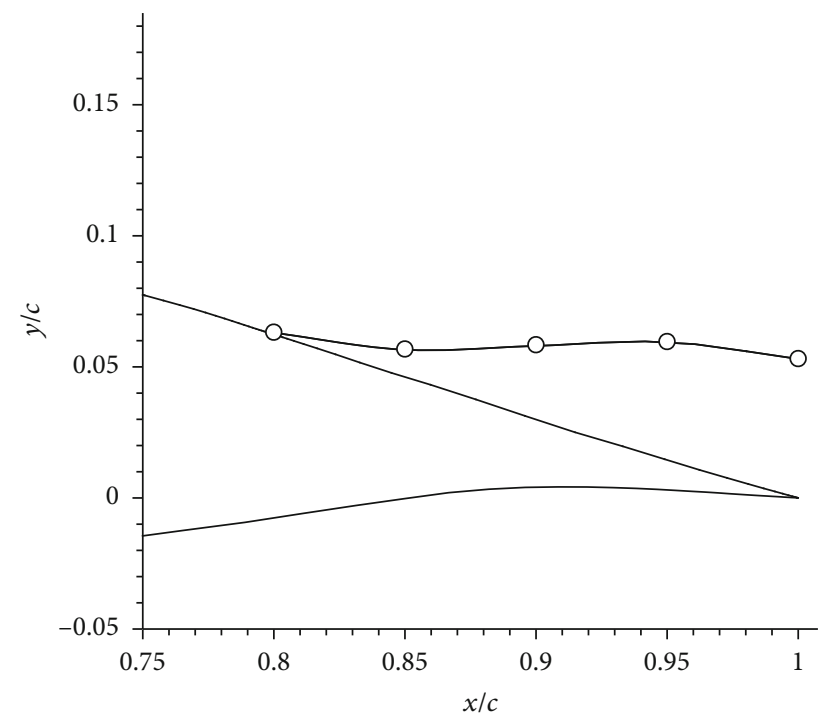

Control point

(c) S2

FIgURE 17: Schematic of the bionic airfoil with three flap shapes. 


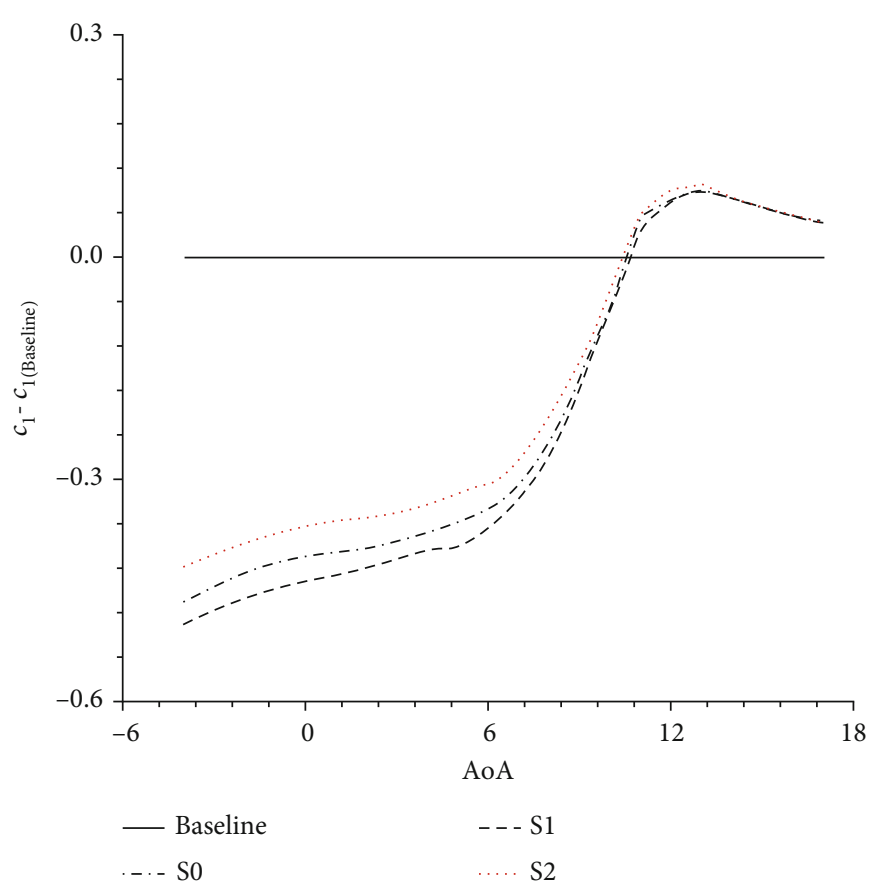

(a) Lift coefficient

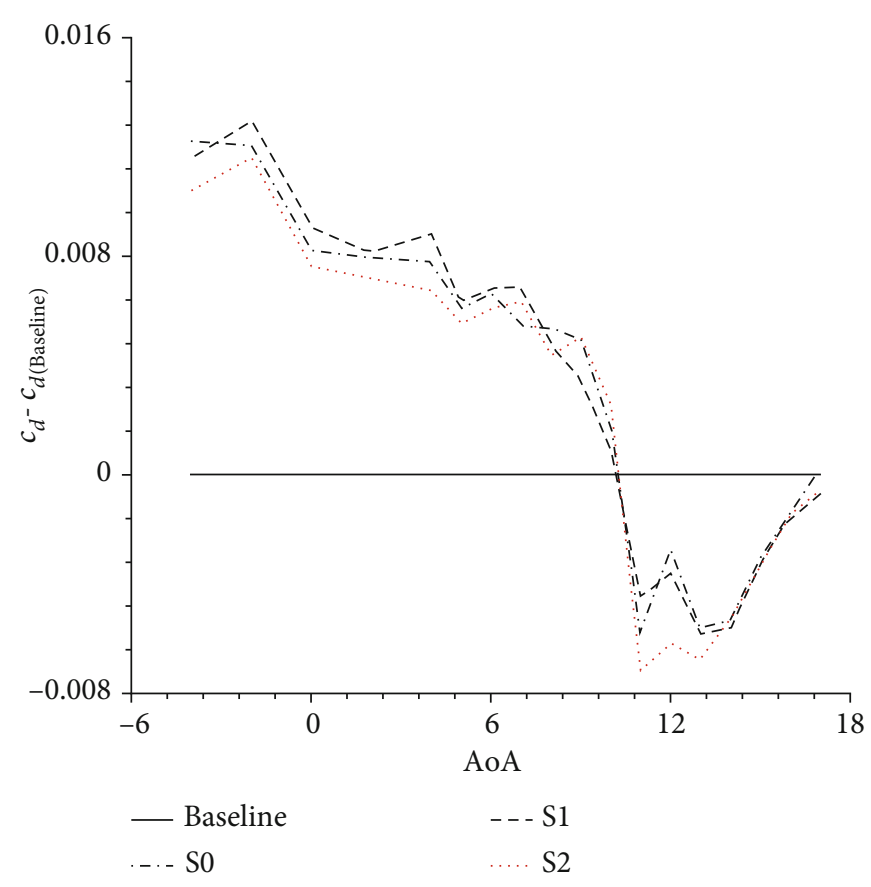

(b) Drag coefficient

FIGURE 18: Variations of the lift and drag coefficients of airfoil with three flap shapes.

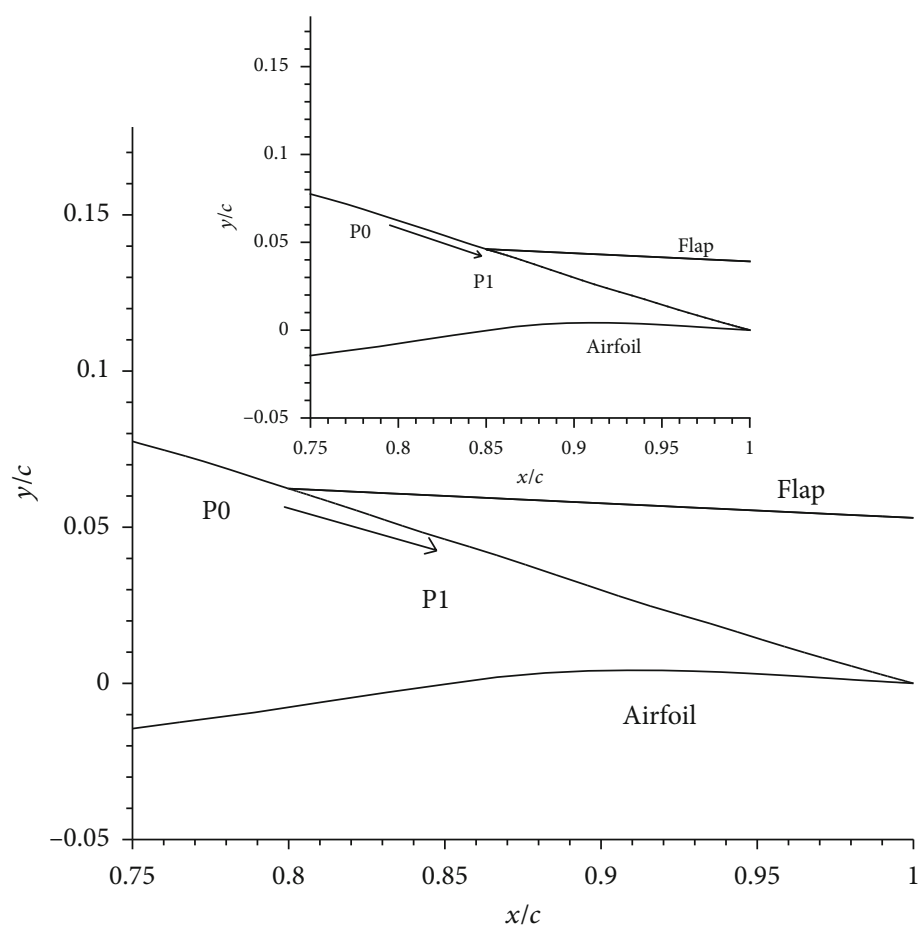

FIGURE 19: Schematic of the bionic airfoil with two flap positions. 


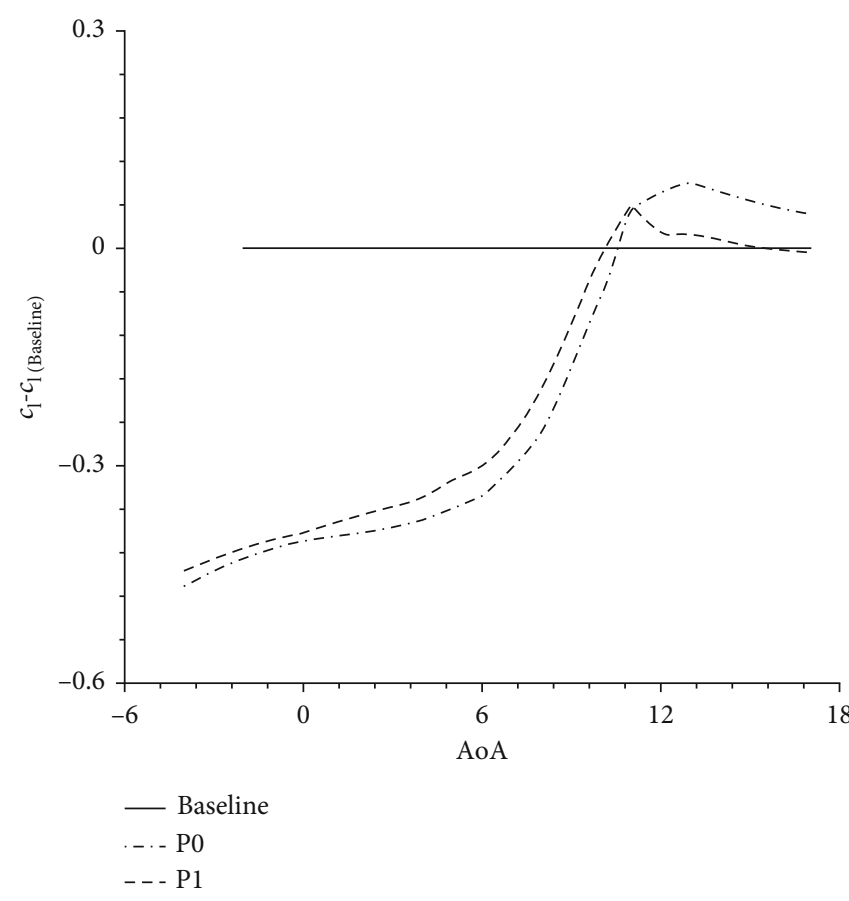

(a) Lift coefficient

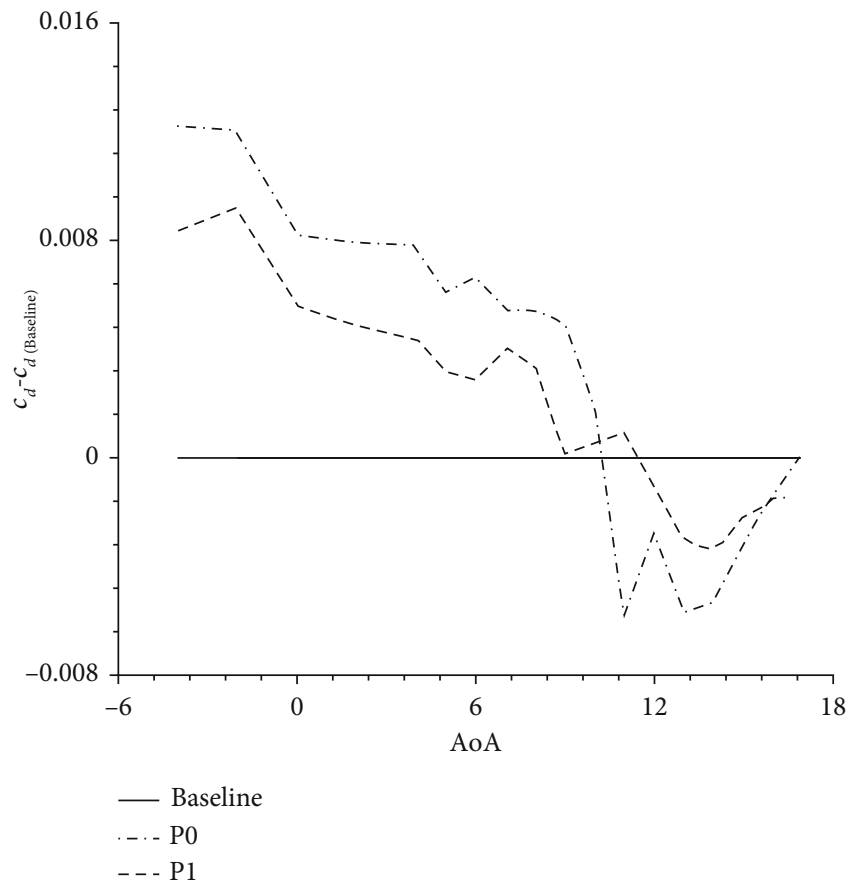

(b) Drag coefficient

Figure 20: Variations of the lift and drag coefficients of airfoil with three flap positions.

TABLE 4: Effect of the flap angles, positions, and shapes on the liftto-drag ratio.

\begin{tabular}{lcccccc}
\hline AoA & $\theta=-2.68^{\circ}(\mathrm{P} 0, \mathrm{~S} 0)$ & $\begin{array}{c}\theta=-0.10 \\
\circ\end{array}$ & $\begin{array}{c}\theta=2.48 \\
\circ\end{array}$ & $\mathrm{P} 1$ & $\mathrm{~S} 1$ & $\mathrm{~S} 2$ \\
\hline 11 & 11.68 & 6.95 & 1.59 & 0.58 & 8.13 & 14.58 \\
12 & 4.57 & 7.27 & 5.02 & 1.37 & 5.39 & 8.82 \\
13 & 5.54 & 6.77 & 7.82 & 2.18 & 5.57 & 6.52 \\
14 & 3.76 & 4.69 & 6.05 & 1.67 & 3.93 & 3.80 \\
15 & 1.98 & 2.52 & 3.22 & 0.74 & 2.04 & 2.08 \\
16 & 1.01 & 1.32 & 1.74 & 0.32 & 1.08 & 1.04 \\
17 & 0.47 & 0.84 & 0.99 & 0.19 & 0.63 & 0.59 \\
\hline
\end{tabular}

\section{Conclusions}

The aerodynamic performance of bionic airfoil with two layouts was studied. The flap angle, shape, and position were the key factors for improving the post stall characteristics of the airfoil. The conclusions were as follows:

(1) The two layouts can significantly improve the stall characteristics of the airfoil. The bionic flaps can suppress the development of flow separation vortex and satisfy the goal of lift enhancement and drag reduction, and the flap with the sinusoid curve shape $\left(\varphi=180^{\circ}\right)$ is the best. The bionic flap angle has a significant effect on the airfoil stall characteristics

(2) As the flap angle increases, the lift decreases significantly at a small AoA; as the lift increases at a large AoA, the stall AoA is also increased. The AoA range for improving the lift and lift-to-drag ratio can reach more than $7^{\circ}$. The excessive flap angle can further increase the maximum lift coefficient, but the AoA range of lift enhancement is smaller

(3) The bionic flap shape has a significant effect on the airfoil stall characteristics

The flaps with the straight line and sinusoid curves with different $\varphi$ were analyzed, and three shapes have almost the same increase in the maximum lift coefficient. For the goal of increasing lift and reducing drag, the sinusoid curve with $\varphi=180^{\circ}$ (S2) is the best, the sinusoid curve shape with $\varphi=0^{\circ}$ (S1) is the worst, and straight line shape (S0) is in the middle

(4) The bionic flap position (gap height) has a significant effect on the airfoil stall

characteristics. The three gap heights have little influence on the lift and drag at small AoA but have significant effect on the stall characteristics. With the increase of gap height, the improvement of stall characteristics is weakened, and the increase of lift and the decrease of drag are both reduced. P0 is the optimal gap height for increasing the lift and liftto-drag ratio

\section{Nomenclature}

$c: \quad$ Chord

$c_{1}:$ Lift coefficient

$c_{\mathrm{d}}$ : Drag coefficient

$c_{\mathrm{p}}$ : Pressure coefficient 
AoA: Angle of attack

$\theta: \quad$ Flap angle

$K$ : $\quad$ Lift-to-drag ratio

$\varphi$ : $\quad$ Phase angle.

\section{Data Availability}

The data used to support the findings of this study were supplied by the corresponding author under license and so cannot be made freely available. Requests for access to these data should be made to the email haolishu@nwpu.edu.cn.

\section{Conflicts of Interest}

The authors declare that there is no conflict of interests regarding the publication of this paper.

\section{Acknowledgments}

The present work is supported by the National Natural Science Foundation of China (11502214) and the Fundamental Research Funds for the Central Universities (D5000200685).

\section{References}

[1] M. A. Aldheeb, W. Asrar, E. Sulaeman, and A. A. Omar, "A review on aerodynamics of non-flapping bird wings," Journal of Aerospace Technology and Management, vol. 8, no. 1, pp. 7-17, 2016.

[2] D. Bechert, M. Bruse, W. Hage et al., "Biological surfaces and their technological application-laboratory and flight experiments on drag reduction and separation control," in 28th Fluid Dynamics Conference, Snowmass Village, CO, USA, 1997.

[3] D. W. Bechert, M. Bruse, W. Hage, and R. Meyer, "Fluid mechanics of biological surfaces and their technological application," Naturwissenschaften, vol. 87, no. 4, pp. 157-171, 2000.

[4] C. B. Liu, Y. J. Wang, L. Q. Ren, and L. Ren, "A review of biological fluid power systems and their potential bionic applications," Journal of Bionic Engineering, vol. 16, no. 3, pp. 367399, 2019.

[5] A. C. Carruthers, S. M. Walker, A. L. R. Thomas, and G. K. Taylor, "Aerodynamics of aerofoil sections measured on a free-flying bird," Proceedings of the Institution of Mechanical Engineers, Part G: Journal of Aerospace Engineering, vol. 224, pp. 855-864, 2010.

[6] T. S. Liu, K. Kuykendoll, R. Rhew, and S. Jones, "Avian wing geometry and kinematics," AIAA Journal, vol. 44, no. 5, pp. 955-963, 2006.

[7] D. Li and X. M. Liu, "Aerodynamic performance and acoustic characteristics of bionic airfoil inspired by three-dimensional long-eared owl wing under low Reynolds number," in Proceedings of ASME Turbo Expo 2016: Turbomachinery Technical Conference and Exposition, Seoul, South Korea, 2016.

[8] S. M. Lim, S. Lee, H. C. Park, K. J. Yoon, and N. S. Goo, "Design and demonstration of a biomimetic wing section using a lightweight piezo-composite actuator (LIPCA)," Smart Materials and Structures, vol. 14, no. 4, pp. 496-503, 2005.

[9] L. D. Peel, J. Megia, B. Narvaez, K. Thompson, and M. Lingala, "Development of a simple morphing wing using elastomeric composites as skins and actuators," Journal of Mechanical Design, vol. 131, no. 9, 2009.
[10] M. Schatz, T. Knacke, F. Thilele, R. Meyer, W. Hage, and D. W. Bechert, "Separation control by self-activated movable flaps," in 42nd AIAA Aerospace Sciences Meeting and Exhibit, Reno, Nevada, USA, 2004.

[11] W. Liebe, "Der auftrieb am tragflügel: entstehung und zusammenbruch," Aerokurier, vol. 12, no. 12, pp. 1520-1523, 1979.

[12] R. K. J. Meyer, Exprimentelle Untersuchungen von Rückstromklappen auf Tragflügeln zur Beeinflussung von Strömungsablösung, [Ph.D. thesis], Technische Universität Berlin, Mensch \& Buch Verlag, Berlin, 2000.

[13] K. H. Kernstine, C. J. Moore, A. Cutler, and R. Mittal, "Initial characterization of self-activated movable flaps, "pop-up feathers"," in 46th AIAA Aerospace Sciences Meeting and Exhibit, Reno, Nevada, USA, 2008.

[14] J. Johnston and A. Gopalarathnam, "Investigation of a bioinspired lift-enhancing effector on a $2 \mathrm{~d}$ airfoil," Bioinspiration \& Biomimetics, vol. 7, no. 3, article 036003, 2012.

[15] G. Allemand and A. Altman, "Post-stall performance improvement through bio-inspired passive covert feathers," in 54th AIAA Aerospace Sciences Meeting and Exhibit, San Diego, California, USA, 2016.

[16] T. S. Liu, J. Montefort, W. Liou, S. R. Pantula, and Q. A. Shams, "Lift enhancement by static extended trailing edge," Journal of Aircraft, vol. 44, no. 6, pp. 1939-1947, 2007.

[17] C. J. Ge, L. Q. Ren, P. Liang, C. C. Zhang, and Z. H. Zhang, "High-lift effect of bionic slat based on owl wing," Journal of Bionic Engineering, vol. 10, no. 4, pp. 456-463, 2013.

[18] M. E. Rosti, M. Omidyeganeh, and A. Pinelli, "Numerical simulation of a passive control of the flow around an aerofoil using a flexible, self adaptive flaplet," Flow Turbulence Combust, vol. 100, no. 4, pp. 1111-1143, 2018.

[19] R. Meyer, W. Hage, D. W. Bechert, M. Schatz, T. Knacke, and F. Thiele, "Separation control by self-activated movable flaps," AIAA Journal, vol. 45, no. 1, pp. 191-199, 2007.

[20] E. Jost, A. Fischer, G. Bangga, T. Lutz, and E. Kramer, "An investigation of unsteady 3-D effects on trailing edge flaps," Wind Energy Science, vol. 2, no. 1, pp. 241-256, 2017.

[21] J. F. Beaudoin and J. L. Aider, "Drag and lift reduction of a 3D bluff body using flaps," Experiments in Fluids, vol. 44, no. 4, pp. 491-501, 2008.

[22] D. Castaignet, T. Barlas, T. Buhl et al., "Full-scale test of trailing edge flaps on a Vestas V27 wind turbine: active load reduction and system identification," Wind Energy, vol. 17, no. 4, pp. 549-564, 2014. 